\title{
Spectral flow, Brouwer degree and Hill's determinant formula
}

\author{
Alessandro Portaluri ${ }^{*} \mathrm{Li} \mathrm{Wu}^{\dagger}$
}

June 2, 2020

\begin{abstract}
In 2005 a new topological invariant defined in terms of the Brouwer degree of a determinant map, was introduced by Musso, Pejsachowicz and the first name author for counting the conjugate points along a semi-Riemannian geodesic. This invariant was defined in terms of a suspension of a complexified family of linear second order Dirichlet boundary value problems.

In this paper, starting from this result, we generalize this invariant to a general self-adjoint Morse-Sturm system and we prove a new spectral flow formula. Finally we discuss the relation between this spectral flow formula and the Hill's determinant formula and we apply this invariant for detecting instability of periodic orbits of a Hamiltonian system.
\end{abstract}

AMS Subject Classification: 58J30, 47H11, 55M25, 58J20.

Keywords: Brouwer degree, Trace formula, Spectral flow, Hill's determinant formula, Elliptic boundary value problems.

\section{Contents}

1 Functional analytic preliminaries $\quad 3$

1.1 Trace class operators: definitions and basics properties . . . . . . . . . . . . . . 3

1.2 Trace class operator valued one-forms . . . . . . . . . . . . . . . . 4

$\begin{array}{lll}2 & \text { An abstract trace formula } & 6\end{array}$

3 Degree index and trace formulas $\quad 11$

3.1 Hill's determinant formula . . . . . . . . . . . . . . . . . . . . . 15

4 Parity of the degree index and instability of periodic orbits $\quad 17$

A A recap on the Maslov index and spectral flow 20

A.1 On the Maslov index . . . . . . . . . . . . . . . . . . . . . . . 20

A.2 On the spectral flow . . . . . . . . . . . . . . . . . . 23

\section{Introduction}

The investigation of the relation between the variational properties of a critical point and geometrical properties of the solution space of its associated linearized equation at this critical point was firstly recognized by Jacobi who proved that the Lagrangian action functional of a regular one dimensional problem is minimized along solutions of the Euler-Lagrange equation up to first conjugate point.

Starting on this result, Morse worked out a precise formula between the Morse index of a critical point of Lagrangian action functional of a regular one dimensional problem and an integer (which somehow generalizes the total number of conjugate points along a geodesic in a Riemannian case)

\footnotetext{
* The author is partially supported by Prin 2015 "Variational methods, with applications to problems in mathematical physics and geometry" No. 2015KB9WPT 001.

${ }^{\dagger}$ The author is supported by NSFC No. $114251 \overline{05}$.
} 
counted along this curve. Since then, many generalizations were obtained to higher order ordinary differential operators, minimal surfaces and elliptic partial differential operators and so on.

The importance of such a relation is due to its central role played in a number of questions related to the dynamical properties of Lagrangian systems as well as in many geometrical problems especially when Duistermaat, in his celebrated and deep paper [Dui76], was able to prove a precise relation between the Morse index and the Maslov index, namely a topological invariant intersection index defined in the Lagrangian Grassmannian manifold.

Starting from Duistermaat aforementioned paper, several different relations were obtained in the last decades and several applications on bifurcation theory to Morse-Floer homology or to stability of periodic orbits of Hamiltonian systems, were proven.

The common denominator of all of these proofs is a sort of spectral flow formula relating the spectral flow (which is the right substitute to the Morse index in the strongly indefinite situation, which usually appears in the Hamiltonian case) with the Maslov (intersection) index.

Roughly speaking, it is well-known, in fact, that the spectral flow of a path of (bounded) selfadjoint Fredholm operators $t \mapsto A_{t}$ is the integer given by the number of negative eigenvalues of $A_{a}$ that become positive as the parameter $t$ goes from $a$ to $b$ minus the number of positive eigenvalues of $A_{a}$ that become negative. By the topological characterization of the space of all bounded selfadjoint Fredholm operators and the definition of the spectral flow it follows that if one of (and hence all) the operators in the path have a finite Morse index, then the spectral flow of a path $A$ is nothing but the difference between the Morse indexes at the end points. Thus spectral flow appears to be the right substitute of the Morse index in the framework of strongly indefinite functionals.

In a series of paper (cfr. [PPT04, MPP05, MPP07, Por09, Por11, PW14c, HP17, HPY19] and references therein), such index theorems were used for detecting the bifurcation along a trivial branch of critical points for family of functionals (maybe in the strongly indefinite situation). Other interesting applications were provided in the last decades for establishing the linear stability and instability properties for critical points of regular and singular Lagrangian system (cfr. [BJP14a, BJP14b, HP19a, HPY19]) or to detect the bifurcation in some elliptic PDE's on bounded domains (cfr. [PW14a, PW14b] and references therein) in which among others authors provided a new proof to the Morse-Smale index theorem [Sma65, Sma67].

Another interesting application of a Morse type index theorem is to graduate the Morse-Floer homology complex (cfr. [APS08] and references therein). In general the spectral flow depends upon the homotopy class of the whole path and not only on its ends. What we propose in this paper is

- a definition of a new topological invariant named index-degree and denoted by $\iota_{\mathrm{PW}}$ defined in terms of a line integral of the trace of an operator-valued one form defined by a suspension of a complexified family of boundary value problems.

- an abstract version of the Morse index theorem which can be summarize as the equality between the spectral flow of a path of self-adjoint unbounded Fredholm operators and $\iota_{\mathrm{PW}}$ which generalize the conjugate index introduced some years ago by author in [MPP05].

As by-product, we provide a different proof to the Morse index theorem proved in [MPP05]. As the reader recognizes, $\iota_{\mathrm{PW}}$-index is very close to other topological invariants which detect gauge anomalies (cf. [Ati84]). Related ideas in the context of Sturm-Liouville boundary value problems can be found in [GST96].

\section{Notation}

At last, for the sake of the reader, let us introduce some common notation that we shall use henceforth without further comments throughout the paper.

- The pair $(H,\langle\cdot, \cdot\rangle)$ will denote a complex separable Hilbert space

- $\mathscr{L}(H)$ (resp. $\left.\mathscr{L}_{s}(H)\right)$ denotes the Banach space of linear bounded (resp. bounded an selfadjoint) operators on $H$ with respect to the operator norm $\|\cdot\|_{\mathscr{L}(H)} ; \mathcal{K}(H)\left(\operatorname{resp} . \mathcal{K}_{s}(H)\right)$ denotes the subspace of $\mathscr{L}(H)$ of compact (resp. compact and symmetric) operators on $H$.

- $S_{1}(H)$ denotes the set of trace class linear operators. 
- Given $A \in \mathscr{L}(H)$ we denote by $A^{*}$ its adjoint. We let $|A|:=\left(A^{*} A\right)^{1 / 2}$.

- $\operatorname{Mat}(N, \mathbb{K})$ denotes the space of $N \times N$ matrices over the field $\mathbb{K}$. $\operatorname{Sym}(N)$ denotes the space of $N \times N$ real symmetric matrices and by $\operatorname{Sym}^{+}(N)$ the set of all positive definite $N \times N$ real symmetric matrices.

- Let $X \subset \mathbb{C}$ be an open subset of the complex plane and let $W$ be a normed vector space. With a slight abuse of notation, we say that $F: X \ni z \mapsto F(z) \in W$ is a $\mathscr{C}^{1}$-map or a map of regularity class $\mathscr{C}^{1}$, if it has regularity class $\mathscr{C}^{1}$ as a map from on open subset of $\mathbb{R}^{2}$ into $W$.

- We denote by $\mathrm{d}$ the exterior differential and finally by $\Omega$ the rectangle defined by

$$
\Omega:=\{z:=t+i s \mid t \in[0,1] \text { and } s \in[-1,1]\} \subseteq \mathbb{C} .
$$

\section{Acknowledgements}

The first name author wishes to thank all faculties and staff of the Mathematics Department at the Shandong University (Jinan) as well as the Department of Mathematics and Statistics at the Queen's University (Kingston) for providing excellent working conditions during his stay and especially his wife, Annalisa, that has been extremely supportive of him throughout this entire period and has made countless sacrifices to help him getting to this point.

\section{Functional analytic preliminaries}

This section is essentially devoted to recall some elementary results that we shall need throughout the paper as well as to introduce our basic notation. In particular we shall discuss some basic properties of trace class operator valued one-forms and we shall prove some technical functional analytic results.

\subsection{Trace class operators: definitions and basics properties}

Let $(H,\langle\cdot, \cdot\rangle)$ be a complex separable Hilbert space, $\mathcal{K}(H)$ be the ideal of compact linear operators on $H$ and let $T \in \mathcal{K}(H)$ and $T^{*}$ its adjoint. It is immediate to check that $T^{*} T$ is a compact self-adjoint non-negative definite linear operator.

For $j \in \mathbb{N}^{*}$, we let $\lambda_{j}\left(T^{*} T\right)$ be the non-zero eigenvalues of $A^{*} A$ where each eigenvalue is repeated according to its own multiplicity and we define the $j$-th singular value or $j$-th $s$ number as the nonnegative number given by $s_{j}(A):=\left[\lambda_{j}\left(T^{*} T\right)^{1 / 2}\right]$.

We set $S_{1}(H):=\left\{T \in \mathcal{K}(H) \mid \sum_{j=1}^{\infty} s_{j}(T)<+\infty\right\}$. Endowing $S_{1}(H)$ with the trace norm given by $\|T\|_{S_{1}}:=\sum_{j=1}^{\infty} s_{j}(T)$, then $S_{1}(H)$ turns into a Banach space, termed the space of trace class operators and its elements are trace class operators on $H$.

REMARK 1.1. We observe that the finite rank operators are dense in $S_{1}(H)$ with respect to the $\|\cdot\|_{S_{1}}$. Moreover $\|T\|_{S_{1}} \geq\|T\|$. (Cf. to [GGK90, Theorem 4.1, Chapter VI, pag.105] for further details). An useful property we shall use throughout the paper is that if $S \in \mathscr{L}(H)$ and $T \in S_{1}(H)$, then $S T \in S_{1}(H)$ and $T S \in S_{1}(H)$.

It is well-known that the trace class $S_{1}(H)$ is a bilateral ideal contained in the ideal of all compact operators $\mathcal{K}(H)$. On $S_{1}(H)$, there is the well-defined linear functional called trace that will be denoted by $\operatorname{Tr}$ and defined by

$$
\operatorname{Tr}: S_{1}(H) \rightarrow \mathbb{R} \quad \text { defined by } \operatorname{Tr}(T):=\sum_{j \in \mathbb{Z}}\left\langle T e_{j}, e_{j}\right\rangle
$$

where $\left(e_{j}\right)_{j \in \mathbb{Z}} \subset H$ is an orthonormal basis of $H$. Among all properties of the trace, we recall that

if $\quad S T, T S \in S_{1}(H) \quad \Rightarrow \quad \operatorname{Tr}(S T)=\operatorname{Tr}(T S)$

and if $\quad S \in S_{1}(H)$ and $T \in \mathscr{L}(H) \quad \Rightarrow \quad\|S T\|_{S_{1}} \leq\|S\|_{S_{1}}\|T\|_{\mathscr{L}(H)}$ and

$$
\|T S\|_{S_{1}} \leq\|S\|_{S_{1}}\|T\|_{\mathscr{L}(H)} .
$$


The next result is the Jacobi's (determinant) formula that we shall need in the proof of the abstract trace forma. Let $z \mapsto S_{z}$ be a $\mathscr{C}^{1}$ map of $N \times N$ complex matrices. Then, the following formula is well-known

$$
\mathrm{d}\left(\operatorname{det}\left(S_{z}\right)\right)=\operatorname{Tr}\left(\operatorname{adj}\left(S_{z}\right) \mathrm{d} S_{z}\right)
$$

where $\operatorname{adj}(\#)$ denotes the adjugate (namely the transpose of the cofactor matrix) of the matrix \#. The following result holds.

Lemma 1.2. Let $S \in \mathscr{C}^{1}(X, \mathrm{GL}(N, \mathbb{C}))$. Then we have

$$
\operatorname{Tr}\left(\mathrm{d} S_{z} S_{z}^{-1}\right)=\mathrm{d} \log \operatorname{det} S_{z} .
$$

Proof. For the proof we refer the interested reader to [MN99] and references therein.

Lemma 1.3. Let $H, L$ be Hilbert spaces, $A \in \mathscr{L}(H, L)$ and let $H_{0}$ be a finite co-dimensional subspace of $H$. If $A_{0}:=\left.A\right|_{H_{0}} \in S_{1}\left(H_{0}\right)$, then $A \in S_{1}(H)$.

Proof. Let $i: H_{0} \mapsto H$ and $j: H_{0}^{\perp} \rightarrow H$ be the injections. Then $i^{*}, j^{*}$ are the orthogonal projections from $H$ to $H_{0}$ and $H_{0}^{\perp}$ respectively. We observe that $\left.A\right|_{H_{0}}=A i$. Since $|A|=i^{*}|A| i+$ $i^{*}|A| j+j^{*}|A| i+j^{*}|A| j$, we have $A_{0}^{*} A_{0}=i^{*} A^{*} A i=\left(i^{*}|A| i\right)^{2}+\left(i^{*}|A| j\right)\left(i^{*}|A| j\right)^{*} \geq\left(i^{*}|A| i\right)^{2}$. Since $A_{0}$ is in the trace class, $i^{*}|A| i$ is also in the trace class and being $H_{0}$ finite co-dimensional subspace of $H,|A|$ is an operator in the trace class. So $\mathrm{A}$ is in the trace class and this concludes the proof.

Lemma 1.4. The Sobolev embedding

$$
\iota: H^{2}\left([0,1], \mathbb{C}^{N}\right) \hookrightarrow L^{2}\left([0,1], \mathbb{C}^{N}\right)
$$

is in the trace class.

Proof. In order to prove that $\iota: H^{2}\left([0,1], \mathbb{C}^{N}\right) \hookrightarrow L^{2}\left([0,1], \mathbb{C}^{N}\right)$ is in the trace class, we start to observe that

$$
\|u\| \mid=\|u\|_{L^{2}}+\left\|D^{2} u\right\|_{L^{2}}
$$

is an equivalent norm in $H^{2}$. Thus we have that the map

$$
D^{2}: H^{2}\left([0,1], \mathbb{C}^{N}\right) \cap H_{0}^{1}\left([0,1], \mathbb{C}^{N}\right) \rightarrow L^{2}\left([0,1], \mathbb{C}^{N}\right)
$$

is an isomorphism. We now observe that $D^{2}: H^{2}\left(I, \mathbb{C}^{N}\right) \cap H_{0}^{1}\left(I, \mathbb{C}^{N}\right) \subset L^{2}\left(I, \mathbb{C}^{N}\right) \rightarrow L^{2}\left(I, \mathbb{C}^{N}\right)$ is in the trace class since it is self-adjoint and the spectrum is given by $\left\{k^{2} \pi^{2}\right\}_{k \in \mathbb{N}}$.

So $\iota H_{H^{2}\left(I, \mathbb{C}^{N}\right) \cap H_{0}^{1}\left(I, \mathbb{C}^{N}\right)}$ is in the trace class. The conclusion now readily follows by using Lemma 1.3 once observed that the space $H^{2}\left([0,1], \mathbb{C}^{N}\right) \cap H_{0}^{1}\left([0,1], \mathbb{C}^{N}\right)$ is a finite-codimensional subspace of $H^{2}\left([0,1], \mathbb{C}^{N}\right)$. This concludes the proof.

\subsection{Trace class operator valued one-forms}

With a slight abuse of notation, given an open subset $X \subset \mathbb{C}$, we let $L \in \mathscr{C}^{1}\left(X, S_{1}(H)\right)$ meaning that $L$ is a a map of regularity class $\mathscr{C}^{1}$ from the open subset of $X \subset \mathbb{R}^{2}$ into $S_{1}(H)$. The next result provides a useful relation between the trace and the exterior differential for an operator valued function. More precisely, the following result holds.

LEMmA 1.5. Let $L \in \mathscr{C}^{1}\left(X, S_{1}(H)\right), F:=I+L$ and we assume that for each $z \in X,\left\|L_{z}\right\|_{\mathscr{L}(H)}<1$. Under the above notation, the following equality holds

$$
\mathrm{d} \operatorname{Tr} \log F_{z}=\operatorname{Tr}\left(\mathrm{d} F_{z} F_{z}^{-1}\right) .
$$

Proof. Since $\left\|L_{z}\right\|_{\mathscr{L}(H)}<1$, then we get that $\log F_{z}=\log \left(I+L_{z}\right)=\sum_{k=1}^{\infty}(-1)^{k+1} \frac{L_{z}^{k}}{k}$ is welldefined. Moreover $\sum_{k=1}^{\infty}(-1)^{k+1} \frac{\operatorname{Tr} L_{z}^{k}}{k}$ converges in $S_{1}(H)$ as directly follows by observing that

$$
\left\|\sum_{k=m}^{n}(-1)^{k+1} \frac{\operatorname{Tr} L_{z}^{k}}{k}\right\|_{S_{1}} \leq\left|\sum_{k=m}^{n}(-1)^{k+1} \frac{\left\|L_{z}\right\|_{\mathscr{L}(H)}^{k-1}}{k}\right|\left\|L_{z}\right\|_{S_{1}(H)} .
$$


By using the linearity of the trace, we get

$$
\operatorname{Tr} \log \left(I+L_{z}\right)=\sum_{k=1}^{\infty}(-1)^{k+1} \frac{\operatorname{Tr} L_{z}^{k}}{k}
$$

and by an induction argument, we get that $\mathrm{d} L_{z}^{k}=\sum_{m=1}^{k} L_{z}^{m-1} \mathrm{~d} L_{z} L_{z}^{k-m}$ for all $z \in X$. Thus, we have

$$
\operatorname{Tr} \mathrm{d} L_{z}^{k}=\sum_{m=1}^{k} \operatorname{Tr}\left[L_{z}^{m-1} \mathrm{~d} L_{z} L_{z}^{k-m}\right]=\sum_{m=1}^{k} \operatorname{Tr}\left[L_{z}^{k-1} \mathrm{~d} L_{z}\right]=k \operatorname{Tr}\left[L_{z}^{k-1} \mathrm{~d} L_{z}\right]=k \operatorname{Tr}\left(\mathrm{d} L_{z} L_{z}^{k-1}\right)
$$

where, in the second and last equality of Equation (2), we used the commutativity property described in Formula (1). By using once again the linearity of Tr and d, we finally get

$$
\begin{aligned}
\mathrm{d} \operatorname{Tr} \log F_{z} & :=\mathrm{d} \operatorname{Tr} \log \left(I+L_{z}\right)=\sum_{k=1}^{\infty}(-1)^{k+1} \frac{\operatorname{Tr} \mathrm{d} L_{z}^{k}}{k} \\
=\sum_{k=1}^{\infty}(-1)^{k+1} \operatorname{Tr}\left(\mathrm{d} L_{z} L_{z}^{k-1}\right)=\operatorname{Tr}\left[\mathrm{d} L_{z} \sum_{k=1}^{\infty}(-1)^{k+1} L_{z}^{k-1}\right]=\operatorname{Tr}\left[\mathrm{d} L_{z}\left(I+L_{z}\right)^{-1}\right] & =\operatorname{Tr}\left(\mathrm{d} F_{z} F_{z}^{-1}\right)
\end{aligned}
$$

where, in the second equality, we commute $d$ and $\sum$ since $\sum_{k=1}^{\infty}(-1)^{k+1} \operatorname{Tr}\left(\mathrm{d} L_{z} L_{z}^{k-1}\right)$ converges uniformly in some neighborhood of $z$ (being, in fact, $z \mapsto L_{z}$ of regularity class $\mathscr{C}^{1}$ in the trace norm topology). This concludes the proof.

LEMma 1.6. Under the assumptions given in Lemma 1.5, we get that the spectrum $\mathfrak{s}\left(F_{z}\right)$ is discrete. Moreover, if $\lambda \in \mathfrak{s}\left(F_{z}\right)$, then for every $\delta>0$, we get $|\lambda-1| \leq \delta$ for all except a finite number.

Proof. By the spectral theory of compact operators, it follows that, for every $z \in X, 0$ is the only accumulation point for eigenvalues of $L_{z}$ and hence 1 is the only possible accumulation point of eigenvalues for $F_{z}$. Thus, for every $\delta>0$ the eigenvalues out off the disk $\{\lambda \in \mathbb{C}|\lambda-1|<\delta\} \subset \mathbb{C}$ counted with multiplicity are in a finite number.

Lemma 1.7. Let $X \subset \mathbb{C}$ be an open and convex set containing $0, W \hookrightarrow H$ be a dense injection and we assume that

- $z \mapsto G_{z} \in \mathscr{L}(W, H)$ is of class $\mathscr{C}^{1}$;

- there is a open set $Y \subset X$ containing 0 and such that $G_{z}$ is invertible for $z \in Y$;

- $z \mapsto\left(G_{z}-G_{0}\right) G_{0}^{-1} \in \mathscr{C}^{1}\left(Y, S_{1}(H)\right)$

Then we get that $\operatorname{Tr} \mathrm{d} G_{z} G_{z}^{-1}$ is a closed one form on $X$.

Furthermore, if $G_{z}=G_{0}+C_{z}$ with $C_{z} \in \mathscr{C}^{1}(X, \mathscr{L}(H))$ and $G_{0}^{-1} \in S_{1}(H)$, then $\operatorname{Tr}\left(\mathrm{d} G_{z} G_{z}^{-1}\right)$ is a closed one form on $Y$.

Proof. For the sake of the reader, we split the proof into some steps.

First step. $\operatorname{Tr} \mathrm{d} G_{z} G_{z}^{-1}$ is a closed one form in a neighborhood of zero.

For, we start to observe that, if $u, v \in W$, then there exists a constant $M>0$ such that

$$
\left|\frac{d}{d t}\left\langle G_{z t} u, v\right\rangle_{H}\right|<M|z|\|u\|_{W}\|v\|_{H} .
$$

By a direct integration on $[0,1]$ it follows that $\left|\left\langle G_{z} u, v\right\rangle-\left\langle G_{0} u, v\right\rangle_{H}\right|<M|z|\|u\|_{W}\|v\|_{H}$ and hence $\left\|G_{z}-G_{0}\right\|_{\mathscr{L}(W, H)} \leq M|z|$. By letting $D_{z}:=G_{z}-G_{0}$, we get that $\left\|D_{z}\right\|_{\mathscr{L}(W, H)} \leq M|z|$. By letting $L_{z}:=D_{z} G_{0}^{-1}=\left(G_{z}-G_{0}\right) G_{0}^{-1}$ and setting $\delta:=\left(2 M\left\|G_{0}^{-1}\right\|_{\mathscr{L}(W, H)}\right)^{-1}$, we get that

$$
\left\|L_{z}\right\|_{\mathscr{L}(H)}=M|z|\left\|G_{0}^{-1}\right\|_{\mathscr{L}(W, H)} \leq M \delta\left\|G_{0}^{-1}\right\|_{\mathscr{L}(W, H)} \leq \frac{1}{2} \quad \text { for }|z| \leq \delta .
$$


Now, observe that $G_{z} G_{0}^{-1}=I+L_{z}$ and the path $z \mapsto I+L_{z}$ is also of class $\mathscr{C}^{1}$. Since $G_{z} G_{0}^{-1}$ is a bijection, we get that $z \mapsto I+L_{z}$ is a path of linear diffeomorphisms. Thus, in particular, the path $z \mapsto\left(I+L_{z}\right)^{-1}$ is a $\mathscr{C}^{1}$ path of bounded operators on $Y$. Then, we have

$$
\begin{aligned}
& \operatorname{Tr}\left[\mathrm{d} G_{z} G_{z}^{-1}\right] \\
& \quad=\operatorname{Tr}\left[\mathrm{d} G_{z}\left(G_{0}\left(I+L_{z}\right)\right)^{-1}\right]=\operatorname{Tr}\left[G_{0} \mathrm{~d} L_{z} G_{0}^{-1}\left(I+L_{z}\right)^{-1}\right]=\operatorname{Tr}\left[\mathrm{d} L_{z}\left(I+L_{z}\right)^{-1}\right] \\
& =\mathrm{d} \operatorname{Tr} \log \left(I+L_{z}\right)
\end{aligned}
$$

where the last equality readily follows by using Lemma 1.5. So $\operatorname{Tr}\left[\mathrm{d} G_{z} G_{z}^{-1}\right]$ is exact in a neighborhood of 0 .

Second step. $\operatorname{Tr} \mathrm{d} G_{z} G_{z}^{-1}$ is a closed one form.

First of all, we observe that by arguing as above, we get that

$$
\left\|G_{z}-G_{z^{\prime}}\right\|_{\mathscr{L}(W, H)} \leq M\left|z-z^{\prime}\right| \quad \forall z, z^{\prime} \in X
$$

and hence also that

$$
\left\|D_{z}-D_{z^{\prime}}\right\|_{\mathscr{L}(W, H)} \leq M\left|z-z^{\prime}\right| \quad \forall z, z^{\prime} \in X
$$

We assume that $z_{0} \in Y$. Then we have

$$
\left(G_{z}-G_{z_{0}}\right) G_{z_{0}}=\left(G_{z}-G_{z_{0}}\right) G_{0}^{-1} G_{0} G_{z_{0}}=\left(L_{z}-L_{z_{0}}\right) G_{0} G_{z_{0}}^{-1}=\left(L_{z}-L_{z_{0}}\right)\left(1+L_{z_{0}}\right)^{-1} .
$$

So, we get that the path $z \mapsto\left(G_{z}-G_{z_{0}}\right) G_{z_{0}}^{-1}$ is a path in $\mathscr{C}^{1}\left(Y, S_{1}(H)\right)$. Since $\left(G_{z}-G_{z_{0}}\right) G_{z_{0}}^{-1} \in$ $S_{1}(H)$, repeating verbatim the argument given in the first step, we also get that for each $z \in Y$, there exists a neighborhood $U_{z}$ such that $\operatorname{Tr}\left[\mathrm{d} G_{z} G_{z}^{-1}\right]$ is exact. Then we get that the form $\operatorname{Tr}\left[\mathrm{d} F_{z} F_{z}^{-1}\right]$ is (globally) closed on $Y$.

The last claim directly follows by the previous one, once observed that, since by assumptions $C_{z} \in \mathscr{C}^{1}(X, \mathscr{L}(H))$ and $G_{0}^{-1} \in S_{1}(H)$, then $z \mapsto\left(G_{z}-G_{0}\right) G_{0}^{-1}=C_{z} G_{0}^{-1}$ is a $\mathscr{C}^{1}$ path in $S_{1}(H)$. This concludes the proof.

\section{An abstract trace formula}

The first result of this subsection, allow us to reduce the computation of the trace of an operatorvalued one form to the sum of the trace of a closed operator-valued one form on a finite dimensional space and the trace of an exact operator-valued one form on a infinite dimensional space. Even if many of the results of this paragraph hold in a more general context, here we shall restrict to the self-adjoint case.

We start by recalling that a bounded perturbation of a compact resolvent operator has compact resolvent as soon as the resolvent set is not empty.

LEMMA 2.1. Let $A_{z_{0}}$ be a self-adjoint operator having compact resolvent and for each $z \in X$ we assume that $C_{z} \in \mathscr{L}(H)$. Then, the operator $A_{z}:=A_{z_{0}}+C_{z}$ has compact resolvent for each $z \in X$.

Proof. This fact can be easily seen as follows. In fact,

$$
\left(\lambda I-A_{z_{0}}\right)-C_{z}=\left[\left(\lambda I-A_{z_{0}}\right)-C_{z}\left(\lambda I-A_{z_{0}}\right)^{-1}\left(\lambda I-A_{z_{0}}\right)\right] .
$$

Since $A_{z_{0}}$ is self-adjoint, there exists $\lambda \in i \mathbb{R}$ such that $\Im(\lambda)>\left\|C_{z}\right\|_{\mathscr{L}(H)}$. We observe that

$$
\left\|C_{z}\left(\lambda I-A_{z_{0}}\right)^{-1}\right\|=\frac{\left\|C_{z}\right\|}{\lambda}\left\|\left(I-\frac{A_{z_{0}}}{\lambda}\right)^{-1}\right\| \leq \frac{\left\|C_{z}\right\|}{\lambda}<1 .
$$

Thus, we get that

$$
\left(\lambda-A_{z_{0}}-C_{z}\right)^{-1}=\left[\left(\lambda I-A_{z_{0}}\right)^{-1}\left(I-C_{z}\left(\lambda I-A_{z_{0}}\right)^{-1}\right)\right] .
$$

Now, the conclusion follows by observing that the first (respectively second) operator at the righthand side of Equation (3) is compact (respectively bounded). This concludes the proof. 
REMARK 2.2. As already observed a general bounded perturbation of a compact(trace class) resolvent operator has compact resolvent if and only if it's resolvent set is not empty!

Now, since the spectrum of a compact resolvent operator consists of isolated points of finite multiplicity, then if $A_{z}$ has compact resolvent then for each $z \in X$ there exists $c>0$ such that

$$
\mathfrak{s}\left(A_{z}\right) \cap S_{c}=\emptyset
$$

where $S_{c}:=\{\lambda \in \mathbb{C}:|\lambda|=c\}$.

We recall that $P_{z}:=-\frac{1}{2 \pi i} \int_{\lambda=c}\left(A_{z}-\lambda I\right)^{-1} \mathrm{~d} \lambda$ is the projection to the total eigenspace corresponding to the eigenvalue of $A_{z}$ in the disk $|\lambda|<c$. We also have $P_{z} A_{z}=A_{z} P_{z}$. We set $Q_{z}:=I-P_{z}$ and we define the following two operators

$$
N_{z}:=Q_{z}+P_{z} A_{z} P_{z} \quad \text { and } \quad M_{z}=P_{z}+Q_{z} A_{z} Q_{z}
$$

By a direct calculation we get that

$$
A_{z}=M_{z} N_{z}=Q_{z} A_{z} Q_{z}+P_{z} A_{z} P_{z}
$$

The following useful results holds.

COROLlaRY 2.3. Under the previous assumptions, the projection $P_{z}$ has finite dimensional range.

Proof. Now, since $A_{z}$ has compact resolvent, it follows that its spectrum consists of finitely many points (counted with their own multiplicity). Moreover, by choosing $\lambda \in \mathbb{C}$ such that $\lambda I-A_{z}$ is invertible it follows that $\mu \rightarrow(\lambda-\mu)^{-1}$ is a one to one map from $\mathfrak{s}\left(A_{z}\right)$ to $\mathfrak{s}\left(\left(\lambda I-A_{z}\right)^{-1}\right)$. Thus, also the set $\left\{z \in \mathbb{C}|| z \mid<c\right.$ and $\left.z \in \mathfrak{s}\left(A_{z}\right)\right\}$ consists of finitely many eigenvalues of $A_{z}$ each of them having finite algebraic multiplicity. So the rank of $P_{z}$ is finite. This concludes the proof.

Lemma 2.4. Let $X \subset \mathbb{C}$ be an open subset, $A_{z_{0}}$ be a operator such that $A_{z_{0}}^{-1} \in S_{1}(H)$ and we let $A_{z}:=A_{z_{0}}+C_{z}$ for $C_{z} \in \mathscr{C}^{1}(X, \mathscr{L}(H))$. Assume that $\mathfrak{s}\left(A_{z_{0}}\right) \cap S_{c}=\emptyset$ for some $c>0$. Then, there exists a (convex) $W_{z_{0}}$ neighborhood of $z_{0}$ such that $A_{z}=M_{z} N_{z}$ for every $z \in W_{z_{0}}$ where $M_{z}$ and $N_{z}$ are given in Equation (4) and $N_{z} \in \mathscr{C}^{1}\left(W_{z_{0}}, \mathscr{L}(H)\right)$.

Proof. Under the previous notations, for $z=z_{0}$, the projection defined by

$$
P_{z}:=-\frac{1}{2 \pi i} \int_{|\lambda|=c}\left(A_{z}-\lambda I\right)^{-1} \mathrm{~d} \lambda
$$

is well-defined and it corresponds to the eigenprojection of $A_{z_{0}}$ for the part of the spectrum contained in the disk $|\lambda|<c$.

Recall that if $T^{-1}$ is bounded invertible, then there is $\delta>0$ such that for each $\|C\|<\delta, T+C$ is bounded invertible and $\left\|(T+C)^{-1}-T^{-1}\right\|<\|C\|\left\|T^{-1}\right\|^{2} /\left(1-\|C\|\left\|T^{-1}\right\|\right)$.

By the compactness of the circle $|\lambda|=c$, we get that there is $\delta>0$ such that $\left(A_{z_{0}}+C_{z}-\lambda I\right)^{-1} \in$ $\mathscr{L}(H)$ for $|\lambda|=c$ with $\left\|C_{z}\right\|<\delta$. So there is a convex $W_{z_{0}}$ neighborhood of $z_{0}$ such that $P_{z}$ is well defined with $z \in W_{z_{0}}$. Note that

$$
P_{z}-P_{z^{\prime}}=-\frac{1}{2 \pi i} \int_{|\lambda|=c}\left(A_{z}-\lambda I\right)^{-1}\left(C_{z^{\prime}}-C_{z}\right)\left(A_{z^{\prime}}-\lambda I\right)^{-1} \mathrm{~d} \lambda .
$$

Since $C_{z} \in \mathscr{C}^{1}(X, \mathscr{L}(H))$, we have $P_{z} \in \mathscr{C}^{1}\left(W_{z_{0}}, \mathscr{L}(H)\right)$. For $z \in W_{z_{0}}$, we also have $P_{z} A_{z} P_{z}=$ $-\frac{1}{2 \pi \mathrm{i}} \int_{\left|\lambda-z_{0}\right|=c} \lambda\left(A_{z}-\lambda I\right)^{-1} \mathrm{~d} \lambda$. Using similarly method above, we have $P_{z} A_{z} P_{z} \in \mathscr{C}^{1}\left(W_{z_{0}}, \mathscr{L}(H)\right)$. Then we can conclude that $N_{z}=Q_{z}+P_{z} A_{z} P_{z} \in\left(W_{z_{0}}, \mathscr{L}(H)\right)$.

Proposition 2.5. Under the assumptions and notation of Lemma 2.4, the following hold

1. $M_{z}=M_{z_{0}}+D_{z}$ with $z \mapsto D_{z} \in \mathscr{C}^{1}\left(W_{z_{0}}, \mathscr{L}(H)\right)$ and $z \mapsto N_{z} \in \mathscr{C}^{1}\left(W_{z_{0}}, \mathscr{L}(H)\right)$;

2. $\operatorname{Tr}\left[\mathrm{d} M_{z} M_{z}^{-1}\right]$ is an exact one-form on $U_{z_{0}}$. 
Let $V_{z_{0}}:=\left\{z \in W_{z_{0}} \mid A_{z}\right.$ invertible $\}$. Then

3. $\operatorname{Tr}\left[\mathrm{d} A_{z} A_{z}^{-1}\right]=\operatorname{Tr}\left[\mathrm{d} M_{z} M_{z}^{-1}\right]+\operatorname{Tr}\left[\mathrm{d} N_{z} N_{z}^{-1}\right]$ on $V_{z_{0}}$

4. $\operatorname{Tr}\left[\mathrm{d} N_{z} N_{z}^{-1}\right]$ is a closed but not exact one-form on $V_{z_{0}}$.

Proof.

As in Lemma 2.4, recall that

$$
P_{z}=-\frac{1}{2 \pi \mathrm{i}} \int_{|\lambda|=c}\left(A_{z}-\lambda I\right)^{-1} \mathrm{~d} \lambda \quad \text { and } \quad P_{z} A_{z} P_{z}=-\frac{1}{2 \pi \mathrm{i}} \int_{|\lambda|=c} \lambda\left(A_{z}-\lambda I\right)^{-1} \mathrm{~d} \lambda .
$$

By using Equation (6), for $z$ in a sufficiently small neighborhood $W_{z_{0}}$ of $z_{0}$ it follows that the path $z \mapsto N_{z}=Q_{z}+P_{z} A_{z} P_{z}$ belongs to $\mathscr{C}^{1}\left(W_{z_{0}}, \mathscr{L}(H)\right)$. By Equation (5), it follows that $Q_{z} A_{z} Q_{z}=A_{z_{0}}+C_{z}-P_{z} A_{z} P_{z}$. We set $D_{z}:=C_{z}-P_{z} A_{z} P_{z}+P_{z}-P_{z_{0}}+P_{z_{0}} A_{z_{0}} P_{z_{0}}$ and we observe that the path $z \mapsto D_{z}$ lies in $\mathscr{C}^{1}\left(W_{z_{0}}, \mathscr{L}(H)\right)$ and moreover $M_{z}=M_{z_{0}}+D_{z}$.

In order to prove the second claim, we observe that for all $z \in U_{z_{0}}$ the operator $M_{z}^{-1}$ is invertible (since 0 is not in the spectrum of $M_{z}$ as easily follows by the definition of $M$ ). Moreover $\mathrm{d} M_{z}=\mathrm{d} D_{z}$ is locally uniformly bounded and $M_{z_{0}}^{-1} \in S_{1}(H)$ (being $M_{z_{0}}$ a bounded perturbation of a trace class resolvent operator $A_{0}$ having not empty resolvent set). By invoking Lemma 1.7, it follows that $\operatorname{Tr}\left[\mathrm{d} M_{z} M_{z}^{-1}\right]$ is an exact one-form on $U_{z_{0}}$.

To prove the third claim, we observe that, since $M_{z}^{-1}=N_{z} A_{z}^{-1}$, then we have

$$
\begin{aligned}
-M_{z}^{-1} \mathrm{~d} M_{z} M_{z}^{-1}=\mathrm{d}\left(M_{z}^{-1}\right)=\mathrm{d} N_{z}\left(A_{z}^{-1}\right)-N_{z} A_{z}^{-1} \mathrm{~d} A_{z} A_{z}^{-1} & \\
& =\mathrm{d} N_{z}\left(N_{z}^{-1} M_{z}^{-1}\right)-N_{z} A_{z}^{-1} \mathrm{~d} A_{z} N_{z}^{-1} M_{z}^{-1} .
\end{aligned}
$$

Since $\operatorname{dom} M_{z}=\operatorname{dom} A_{z}$ is dense in $H$, we get by the first and last members of Equation $(7)$, that

$$
M_{z}^{-1} \mathrm{~d} M_{z}=-\mathrm{d} N_{z} N_{z}^{-1}+N_{z} A_{z}^{-1} \mathrm{~d} A_{z} N_{z}^{-1}
$$

and by using once again the commutativity property of the trace, we also get that

$$
\operatorname{Tr} \mathrm{d} A_{z} A_{z}^{-1}=\operatorname{Tr} \mathrm{d} M_{z} M_{z}^{-1}+\operatorname{Tr} \mathrm{d} N_{z} N_{z}^{-1} .
$$

In order to prove the fourth claim, we observe that by Lemma 1.7, the one-form $\operatorname{Tr}\left(\mathrm{d} A_{z} A_{z}^{-1}\right)$ is closed on $V_{z_{0}}$. Then by the claim 3 . we get that $\operatorname{Tr}\left[\mathrm{d} N_{z} N_{z}^{-1}\right]$ is also closed. This concludes the proof.

Proposition 2.6. Given $\varepsilon>0$, let $A \in \mathscr{C}^{1}((-\varepsilon, \varepsilon), \mathrm{GL}(W, H))$ be a path of invertible operators on a Hilbert space $H$ having the same domain $W$ and let $P \in \mathscr{C}^{1}((-\varepsilon, \varepsilon), \mathscr{L}(H))$ be a path of finite rank projections. We assume that

- $P_{t} A_{t}=A_{t} P_{t}$ for all $t \in(-\varepsilon, \varepsilon)$

- $t \mapsto P_{t} A_{t} P_{t} \in \mathscr{C}^{1}((-\epsilon, \epsilon), \mathscr{L}(H))$

Then we get that $E_{t}:=\left.P_{t} A_{t} P_{t}\right|_{\operatorname{Im} P_{t}}$ is a linear map from $\operatorname{Im} P_{t}$ to $\operatorname{Im} P_{t}$ and by setting $N_{t}:=$ $\left(I-P_{t}\right)+P_{t} A_{t} P_{t}$, the following equality holds:

$$
\operatorname{Tr}\left[\mathrm{d} N_{t} N_{t}^{-1}\right]=\operatorname{Tr}\left[\mathrm{d}\left(P_{t} A_{t} P_{t}\right) P_{t} A_{t}^{-1} P_{t}\right]=\mathrm{d} \log \operatorname{det} E_{t} .
$$

Proof. Without loss of generality, we only need to prove the equality at $t=0$; thus we assume that $\operatorname{dim} \operatorname{Im} P_{0}=n$ and let $\left(e_{i}\right)_{i=1}^{n}$ be a basis of $\operatorname{Im} P_{0}$. Then for $|t|$ sufficiently small, we get $\left\{P_{t} e_{1}, P_{t} e_{2}, \cdots, P_{t} e_{n}\right\}$ is a basis of $\operatorname{Im} P_{t}$. Let $P_{t} A_{t} P_{t} e_{i}=\sum_{1 \leq j \leq n} \alpha_{i j}(t) P_{t}\left(e_{j}\right)$. Then we have $E_{t}\left(P_{t} e_{j}\right)=\sum_{1 \leq j \leq n} \alpha_{i j}(t) P_{t} e_{j}$. Furthermore

$$
P_{0} P_{t} A_{t} P_{t} P_{0} e_{i}=\sum_{1 \leq j \leq n} \alpha_{i j}(t) P_{0} P_{t} P_{0} e_{j}
$$


Let now $R_{t}:=P_{0} P_{t} P_{0}$ be the map from $\operatorname{Im} P_{0}$ to $\operatorname{Im} P_{0}$ and we let $R_{t} e_{i}=\sum_{1 \leq j \leq n} \beta_{i j}(t) e_{j}$. We denote by $S_{t}$ the map from $\operatorname{Im} P_{0}$ to $\operatorname{Im} P_{0}$ defined by $S_{t}:=P_{0} P_{t} A_{t} P_{t} P_{0}$. Then we have

$$
S_{t} e_{i}=\sum_{1 \leq j, k \leq n} \alpha_{i j}(t) \beta_{j k}(t) e_{k} .
$$

By Lemma 1.2 and by Equation (8), we get

$$
\left.\operatorname{Tr}\left[\mathrm{d} S_{t} S_{0}^{-1}\right]\right|_{t=0}=\left.\mathrm{d} \log \operatorname{det} S_{t}\right|_{t=0}=\left.\mathrm{d} \log \operatorname{det} E_{t}\right|_{t=0}+\left.\mathrm{d} \log \operatorname{det} R_{t}\right|_{t=0} .
$$

We note that $\left.\mathrm{d} S_{t}\right|_{t=0} S_{0}^{-1}=\left.P_{0} \mathrm{~d}\left(P_{t} A_{t} P_{t}\right)\right|_{t=0} P_{0}\left(P_{0} A_{0}^{-1} P_{0}\right)$ and hence

$$
\left.\operatorname{Tr}\left[\mathrm{d} S_{t} S_{0}^{-1}\right]\right|_{t=0}=\operatorname{Tr}\left[\left.\mathrm{d}\left(P_{t} A_{t} P_{t}\right)\right|_{t=0} P_{0} A_{0}^{-1} P_{0}\right] .
$$

By invoking once again Lemma 1.2, we also have

$$
\left.\mathrm{d} \log \operatorname{det} R_{t}\right|_{t=0}=\operatorname{Tr}\left[\left.\mathrm{d}\left(P_{0} P_{t} P_{0}\right)\right|_{t=0} P_{0}\right]=\operatorname{Tr}\left[\left.P_{0} \mathrm{~d} P_{t}\right|_{t=0}\right]=\left.\frac{1}{2} \operatorname{Tr} \mathrm{d} P_{t}^{2}\right|_{t=0}=\left.\frac{1}{2} \operatorname{Tr} \mathrm{d} P_{t}\right|_{t=0} .
$$

We note that $\operatorname{Tr}\left(P_{t} \mathrm{~d} P_{t}\right)=\operatorname{Tr}\left(P_{t} \mathrm{~d} P_{t}^{2}\right)=\operatorname{Tr}\left(P_{t} P_{t} \mathrm{~d} P_{t}+P_{t} \mathrm{~d} P_{t} P_{t}\right)=2 \operatorname{Tr}\left(P_{t} \mathrm{~d} P_{t}\right)$. Then we have

$$
\operatorname{Tr}\left(\mathrm{d} P_{t}\right)=2 \operatorname{Tr}\left(P_{t} \mathrm{~d} P_{t}\right)=0 .
$$

Then we can conclude that

$$
\operatorname{Tr}\left[\left.\mathrm{d}\left(P_{t} A_{t} P_{t}\right)\right|_{t=0} P_{0} A_{0}^{-1} P_{0}\right]=\left.\mathrm{d} \log \operatorname{det} E_{t}\right|_{t=0}
$$

By arguing as above, for each $t \in(-\epsilon, \epsilon)$, we get

$$
\operatorname{Tr}\left[\mathrm{d}\left(P_{t} A_{t} P_{t}\right) P_{t} A_{t}^{-1} P_{t}\right]=\mathrm{d} \log \operatorname{det} E_{t} .
$$

Let us consider the projection $Q_{t}=I-P_{t}$ and we observe that

$$
\operatorname{Tr}\left[\mathrm{d} N_{t} N_{t}^{-1}\right]=\operatorname{Tr}\left[\left(\mathrm{d} Q_{t}+\mathrm{d}\left(P_{t} A_{t} P_{t}\right)\right)\left(Q_{t}+P_{t} A_{t}^{-1} P_{t}\right)\right] .
$$

We observe that

$$
\mathrm{d}\left(P_{t} A_{t} P_{t}\right)=\mathrm{d}\left(P_{t} A_{t} P_{t}^{2}\right)=d\left(P_{t} A_{t} P_{t}\right) P_{t}+P_{t} A_{t} P_{t} d P_{t}
$$

and by the first and third member in the previous equation, we get that $\mathrm{d}\left(P_{t} A_{t} P_{t}\right) Q_{t}=P_{t} A_{t} P_{t} d P_{t}$. By this, it follows that $\operatorname{Tr}\left(\mathrm{d}\left(P_{t} A_{t} P_{t}\right) Q_{t}\right)=0$.

Similarly we have

$$
\operatorname{Tr}\left[\mathrm{d} Q_{t}\left(P_{t} A_{t}^{-1} P_{t}\right)\right]=\operatorname{Tr}\left[\mathrm{d} Q_{t}^{2}\left(P_{t} A_{t}^{-1} P_{t}\right)\right]=\operatorname{Tr}\left[Q_{t} \mathrm{~d} Q_{t} P_{t} A_{t}^{-1} P_{t}+\mathrm{d} Q_{t} Q_{t}\left(P_{t} A_{t}^{-1} P_{t}\right)\right]=0 .
$$

Then we can conclude that $\operatorname{Tr}\left(\mathrm{d} N_{t} N_{t}^{-1}\right)=\operatorname{Tr}\left[\mathrm{d}\left(P_{t} A_{t} P_{t}\right) P_{t} A_{t}^{-1} P_{t}\right]$. Summing up this last equation and Equation (9), we get the desired conclusion.

For, let us consider the family of operators parametrized by the rectangle $\Omega:=[0,1] \times[-1,1] \subset \mathbb{C}$

$$
A_{z}:=A_{t}+i s I \quad \text { where } \quad z:=t+i s \in \Omega
$$

where $A_{t}$ is self-adjoint for every $t$. Arguing preciely as in Proposition 2.6, we assume that there is a path of finite rank projections $P \in \mathscr{C}^{1}([0,1], \mathscr{L}(H))$ such that $P_{t} A_{t}=A_{t} P_{t}$ and $t \mapsto P_{t} A_{t} P_{t} \in$ $\mathscr{C}^{1}([0,1], \mathscr{L}(H))$. Let $S_{z}:=\left.\left[P_{t} A_{t} P_{t}+i s I\right]\right|_{\operatorname{Im} P_{t}}$ and we recall that there exist $n$ continuous functions that represent the repeated eigenvalues of $\left.P_{t} A_{t} P_{t}\right|_{\operatorname{Im} P_{t}}$ and, up to relabel, we can assume that

$$
\lambda_{1}(t) \leq \ldots \leq \lambda_{n}(t) .
$$

Thus, we have $\operatorname{det} S_{z}=\prod_{i=1}^{n}\left[\lambda_{i}(t)+i s\right]$. 
Proposition 2.7. Under the above notation and assume that $A_{0}, A_{1}$ is bounded invertible, $A_{0}^{-1} \in$ $S_{1}(H)$, and $A_{t}=A_{0}+C_{t}$ with $C_{t} \in \mathscr{C}^{1}\left([0,1], \mathscr{L}_{s}(H)\right)$. We assume that there is $c>0$ such that $\pm c \notin \mathfrak{s}\left(A_{t}\right)$, for each $t \in[0,1]$. Then the decomposition $A_{t}=M_{t} N_{t}$ is well defined on $[0,1]$ and we have

$$
\frac{1}{2 \pi i} \int_{\partial \Omega} \operatorname{Tr} \mathrm{d} A_{z} A_{z}^{-1}=l-m
$$

where $l$ (respectively $m$ ) is the number of $\lambda_{i}(t)$ (counted with multiplicity) crossing the real axis from negative to positive (respectively from positive to negative).

Proof. The proof of this result is quite straightforward. First, we observe that on $\Omega$ the following decomposition holds

$$
A_{z}=M_{z} N_{z} \text {, where } M_{z}=P_{t}+Q_{t}\left(A_{t}+i s I\right) Q_{t} \text {, and } N_{z}=Q_{t}+P_{t}\left(A_{t}+i s I\right) P_{t} .
$$

By invoking Proposition 2.5, we get

$$
\frac{1}{2 \pi i} \int_{\partial \Omega} \operatorname{Tr} \mathrm{d} A_{z} A_{z}^{-1}=\frac{1}{2 \pi i} \int_{\partial \Omega} \operatorname{Tr} \mathrm{d} N_{z} N_{z}^{-1}+\frac{1}{2 \pi i} \int_{\partial \Omega} \operatorname{Tr} \mathrm{d} M_{z} M_{z}^{-1}=\frac{1}{2 \pi i} \int_{\partial \Omega} \operatorname{Tr} \mathrm{d} N_{z} N_{z}^{-1} .
$$

By invoking Proposition 2.6, we get

$$
\frac{1}{2 \pi i} \int_{\partial \Omega} \operatorname{Tr} \mathrm{d} N_{z} N_{z}^{-1}=\left.\frac{1}{2 \pi i} \int_{\partial \Omega} \mathrm{d} \log \operatorname{det}\left[P_{t} A_{t} P_{t}+i s I\right]\right|_{\operatorname{Im} P_{t}} .
$$

Let $S_{z}:=\left.\left[P_{t} A_{t} P_{t}+i s I\right]\right|_{\operatorname{Im} P_{t}}$. There exist $n$ continuous functions that represent the repeated eigenvalues of $P_{t} A_{t} P_{t}$ and, up to relabel, we can assume that

$$
\lambda_{1}(t) \leq \ldots \leq \lambda_{n}(t)
$$

Thus, we have $\operatorname{det} S_{z}=\prod_{i=1}^{n}\left[\lambda_{i}(t)+i s\right]$. So, let us consider the eigenvalue $\lambda_{i}$ and we observe that only four cases can occur (according to the sign of $\lambda_{i}$ at the ends); more precisely
(I) $\left\{\begin{array}{l}\lambda_{i}(0)<0 \\ \lambda_{i}(1)>0\end{array}\right.$
(II) $\left\{\begin{array}{l}\lambda_{i}(0)>0 \\ \lambda_{i}(1)<0\end{array}\right.$,
$(I I I)\left\{\begin{array}{l}\lambda_{i}(0)<0 \\ \lambda_{i}(1)<0\end{array}\right.$
$(I V)\left\{\begin{array}{l}\lambda_{i}(0)>0 \\ \lambda_{i}(1)>0\end{array}\right.$

For each one of the cases appearing in Equation (10), we construct the following four homotopies

$$
\begin{aligned}
h_{(I)}(s, t):=(1-s) \lambda_{i}(t)+s\left(t-\frac{1}{2}\right), \quad & h_{(I I)}(s, t):=(1+s) \lambda_{i}(t)+s\left(-t+\frac{1}{2}\right) \\
& h_{(I I I)}(s, t):=(1-s) \lambda_{i}(t)-s \quad h_{(I V)}(s, t):=(1-s) \lambda_{i}(t)+s, .
\end{aligned}
$$

Thus, there exist $h, k, l, m \in \mathbb{N}$ such that $\operatorname{det} S_{z}$ is a homotopic (through an admissible homotopy) to the following function

$$
\phi(t, s):=(1+i s)^{k}(-1+i s)^{h}\left(t-\frac{1}{2}+i s\right)^{l}\left(-t+\frac{1}{2}+i s\right)^{m}
$$

where $n=h+k+l+m$. Moreover the functions $s \mapsto 1+i s$ and $s \mapsto-1+i s$ are homotopic to the constant functions 1 and -1 respectively through the homotopy $s \mapsto 1+i \lambda s$ and $s \mapsto-1+i \lambda s$, for $\lambda \in[0,1]$ respectively. Thus, we have

$$
\begin{gathered}
\operatorname{deg}\left(\operatorname{det} S_{z}, R, 0\right) \\
=\frac{1}{2 \pi i} \int_{\partial R} \mathrm{~d} \log \operatorname{det} S_{z}=\frac{1}{2 \pi i} \int_{\partial R} \mathrm{~d} \log \left[\left(t-\frac{1}{2}+i s\right)^{l}\left(-t+\frac{1}{2}+i s\right)^{m}\right] \\
\quad=\frac{1}{2 \pi i} \int_{\partial R} \mathrm{~d} \log \left(t-\frac{1}{2}+i s\right)^{l}+\frac{1}{2 \pi i} \int_{\partial R} \mathrm{~d} \log \left(-t+\frac{1}{2}+i s\right)^{m}
\end{gathered}
$$

$$
=l-m
$$

This concludes the proof.

REMARK 2.8. The proof given in Proposition 2.7 mainly relies on the homotopy invariance property of the winding number and it is completely differen of a similar result proved by authors in [MPP05, Proposition 5.1] by using Kato's Selection Theorem. 


\section{Degree index and trace formulas}

This section is devoted to introduce a new invariant defined by means of a suspension of the complexified family of Morse-Sturm boundary value problems. The resulting boundary value problem is parameterized by points of the complex plane. This topological invariant that will be defined in terms of the Brouwer degree of an associated determinant map.

We consider a linear second order differential operator

$$
\mathscr{A}_{0}:=-\frac{d}{d x}\left[P(x) \frac{d}{d x}+Q(x)\right]+Q^{\top}(x) \frac{d}{d x}+G(x) \quad x \in[0,1]
$$

with matrix coefficients $P, G \in \mathscr{C}^{1}([0,1], \operatorname{Sym}(N)), Q \in \mathscr{C}^{1}([0,1], \operatorname{Mat}(N, \mathbb{R}))$ and we assume that $P(x)$ is non degenerate for each $x \in[0,1]$.

Now, for every $t \in[0,1]$, we let $C_{t} \in \mathscr{C}^{1}(I, \operatorname{Sym}(N))$ and we assume that $t \mapsto C_{t}(x)$ is continuous and $C_{0}(x)=0$.

We set $C_{1}(x)=C(x)$ and let us now define the second order differential operator

$$
\mathscr{A}_{t}:=\mathscr{A}_{0}+\mathscr{C}_{t}
$$

where $\mathscr{C}_{t}$ denotes the operator pointwise defined by $C_{t}$ as follows $\left(\mathscr{C}_{t} u\right)(x):=C_{t} u(x)$ for every $x \in[0,1]$. Without further conditions, the operators $\mathscr{A}_{0}$ and hence $\mathscr{A}_{t}$ acts, for each $t \in[0,1]$ on the space $\mathscr{C}^{1}\left([0,1], \mathbb{R}^{N}\right)$.

Definition 3.1. For $i=0,1$ we set $R_{i} \in \operatorname{Mat}(2 N, \mathbb{R})$ and we define the boundary operator $\mathcal{R}$ as

$$
\mathcal{R}(u):=R_{0}\left[\begin{array}{c}
P(0) u^{\prime}(0)+Q(0) \\
u(0)
\end{array}\right]+R_{1}\left[\begin{array}{c}
P(1) u^{\prime}(1)+Q(1) \\
u(1)
\end{array}\right]
$$

where' denotes the derivative with respect to $x$.

ExAmple 3.2. We observe that the boundary conditions given in Definition 3.1 are very general. It is worth noticing that:

- Dirichlet case corresponds to choose

$$
R_{0}:=\left[\begin{array}{cc}
0 & I_{n} \\
0 & 0
\end{array}\right], \quad R_{1}:=\left[\begin{array}{cc}
0 & 0 \\
0 & I_{n}
\end{array}\right] ;
$$

- Neumann case corresponds to choose

$$
R_{0}:=\left[\begin{array}{cc}
I_{n} & 0 \\
0 & 0
\end{array}\right], \quad R_{1}:=\left[\begin{array}{cc}
0 & 0 \\
I_{n} & 0
\end{array}\right]
$$

- Periodic boundary conditions corresponds to choose

$$
R_{0}=\left[\begin{array}{cc}
I & 0 \\
0 & I
\end{array}\right], \quad R_{1}=-R_{0} .
$$

For each $t \in I$, we denote by $\mathcal{A}_{0}$ and $\mathcal{A}_{t}$, the operator $\mathscr{A}_{0}$ and $\mathscr{A}_{t}$ respectively acting on the domain $\mathcal{D}:=\left\{u \in H^{2}\left(I, \mathbb{R}^{N}\right) \mid \mathcal{R} u=0\right\}$ and we consider the complexified (extension of the) operators $\mathcal{A}_{0}$ and $\mathcal{A}_{t}$ by considering both operators acting on the $\mathrm{C}^{\infty}\left(I, \mathbb{C}^{N}\right)$. With a slight abuse of notation we will not distinguish between $\mathcal{D}$ and its complexification

$$
\mathcal{D}:=\left\{u \in H^{2}\left(I, \mathbb{C}^{N}\right) \mid \mathcal{R} u=0\right\} .
$$

as well as between $\mathcal{A}_{0}$ and $\mathcal{A}_{t}$ and their complex extensions. We let

$$
C_{z}(x):=C_{t}(x)+i s I \quad \text { for } \quad z=t+i s \in \Omega
$$

and for every $z \in \Omega$ we define $\mathcal{A}_{z}: \mathcal{D} \subset L^{2}\left([0,1], \mathbb{C}^{N}\right) \rightarrow L^{2}\left([0,1], \mathbb{C}^{N}\right)$ to be the closed unbounded operator on $\mathcal{D}$ pointwise defined by

$$
\left(\mathcal{A}_{z} u\right)(x):=\left(\mathcal{A}_{0} u\right)(x)+\mathcal{C}_{z}(x) .
$$


We consider the Morse-Sturm equation

$$
-\frac{d}{d x}\left[P(x) u^{\prime}(x)+Q(x) u(x)\right]+Q^{\top}(x) u^{\prime}(x)+G(x) u(x)+C_{z}(x) u(x)=0, \quad x \in[0,1] .
$$

By setting $v(x):=P(x) u^{\prime}(x)+Q(x) u(x)$ and $w(x) \equiv(v(x), u(x))^{\top}$, Equation (11) fits into the following linear Hamiltonian system

$$
w^{\prime}(x)=J B_{z}(x) w(x), \quad x \in[0,1]
$$

where $J:=\left[\begin{array}{cc}0 & -I \\ I & 0\end{array}\right]$ and finally

$$
B_{z}(x):=\left[\begin{array}{cc}
P^{-1}(x) & -P^{-1}(x) Q(x) \\
-Q^{\top}(x) P^{-1}(x) & Q^{\top}(x) P^{-1}(x) Q(x)-G(x)-C_{z}(x)
\end{array}\right] .
$$

Let $\psi_{z}: I \rightarrow \mathrm{Sp}(2 N)$ be the fundamental solution of the Hamiltonian system given in Equation (12) and we let

$$
R_{z}:=R_{0}+R_{1} \psi_{z}(1), \quad z \in \Omega .
$$

LEMMA 3.3. The following statements are equivalent

1. $\operatorname{ker} \mathcal{A}_{z} \neq\{0\}$

2. $\operatorname{det} R_{z}=0$

Setting $z:=\left\{z \in \Omega \mid \operatorname{det} R_{z}=0\right\}$ we have that

$$
z \subset \mathbb{R}
$$

Proof. $(\Rightarrow)$ Let $u \in \operatorname{ker} \mathcal{A}_{z}$ and let $w$ be a solution of Equation (12). Thus $w(1)=\psi_{z}(1) w(0)$ and $R_{z} w(0)=R_{0} w(0)+R_{1} \psi_{z}(1) w(0)=R_{0} w(0)+R_{1} w(1)$ and by this we get that $R_{z} w(0)=0$. So now, we assume by contradiction that $\operatorname{det} R_{z} \neq\{0\}$ then we have $w(0)=0$ which in particularly means that $u(0)=u^{\prime}(0)=0$ (being $P(x)$ non-degenerate for every $x \in[0,1]$ ). Thus by the existence and uniqueness theorem for first order linear differential equations, we get that $w \equiv 0$. Thus $\operatorname{ker} \mathcal{A}_{z}=\{0\}$ which is a contradiction. This concludes the proof of the only if part.

$(\Leftarrow)$ To prove the if part we start to observe that since $\operatorname{det} R_{z}=\{0\}$, then there exists a non-trivial $w_{0} \in \operatorname{ker} R_{z}$. We set $w(x)=\psi_{z}(x) w_{0}$ and we observe that $w$ is a solution of Equation (12) and satisfies the boundary condition. It is immediate to check that, if $\mathcal{P}_{z}: \mathbb{R}^{2 N} \rightarrow \mathbb{R}^{N} \oplus \mathbb{R}^{N}$ is the projection onto the second component, then $u(x)=\mathcal{P}_{z} w(x)$ lies in $\operatorname{ker} A_{z}$.

In order to prove the last statement, it is enough to observe that the spectrum of a self-adjoint operator is real; thus $\operatorname{ker} A_{z} \neq\{0\}$ can occur only at $z=t+i s$ with $s=0$. This concludes the proof.

DEFINITION 3.4. Under the notation above, we define the determinant map $\rho$ as follows:

$$
\rho: \Omega \ni z \longmapsto \rho(z):=\operatorname{det} \mathcal{R}_{z} \in \mathbb{C} .
$$

We assume that $\mathcal{A}_{0}$ and $\mathcal{A}_{1}$ are non-degenerate meaning that $\operatorname{ker} \mathcal{A}_{0}=\operatorname{ker} \mathcal{A}_{1}=\{0\}$. In shorthand notation we simply refer to $t \mapsto \mathcal{A}_{t}$ or simply $\mathcal{A}$ as admissible. Thus in particular, by Lemma 3.3, we get that $0 \notin \rho(\partial \Omega)$.

DEFINITION 3.5. Under the previous notation, if $0 \notin \rho(\partial \Omega)$, we define the degree index associated to the pair $(\psi, R)$ as the integer $\iota_{\mathrm{PW}}(\psi, R)$ defined by

$$
\iota_{\mathrm{PW}}(\psi, R):=\operatorname{deg}(\rho, \Omega, 0) .
$$

We term spectral index of $\mathcal{A}$ the integer $\iota_{\mathrm{SP}}(\mathcal{A})$ given by

$$
\iota_{\mathrm{SP}}(\mathcal{A}):=\operatorname{sf}(\mathcal{A},[0,1]) .
$$


REMARK 3.6. It is worth noticing that the Brouwer degree of a map $\rho$ on the open set $\Omega$ is related to the winding number as follows

$$
\operatorname{deg}(\rho, \Omega, 0)=\frac{1}{2 \pi i} \int_{\partial \Omega} \mathrm{d} \log \rho_{z} .
$$

Now, to the family $\mathcal{A}_{z}$ defined at Equation (11), we associate the operator valued one-form defined by $\Theta_{z}:=\mathrm{d} \mathcal{A}_{z} \mathcal{A}_{z}^{-1}$. More explicitly

$$
\Theta_{z}:=\mathrm{d} \mathcal{A}_{z} \mathcal{A}_{z}^{-1}=\partial_{t} \mathfrak{C}_{z} \mathcal{A}_{z}^{-1} \mathrm{~d} t+\partial_{s} \mathcal{C}_{z} \mathcal{A}_{z}^{-1} \mathrm{~d} s=\partial_{t} \mathcal{C}_{t} \mathcal{A}_{z}^{-1} \mathrm{~d} t+i \mathcal{A}_{z}^{-1} \mathrm{~d} s
$$

defined on the subset $\left\{z \in \Omega \mid \mathcal{A}_{z}\right.$ has a bounded inverse $\}$.

By invoking Lemma 3.3 and [GGK90, Theorem 3.1, pag. 295-296], $\mathcal{A}_{z}$ has a bounded inverse on $\partial \Omega$. Moreover $\mathcal{A}_{z}^{-1}$ is a Hilbert-Schmidt operator. In fact, we have

$$
\mathcal{A}_{z}^{-1} u(x)=\int_{0}^{1} K_{z}(x, y) u(y) d y
$$

where the Green's kernel is given by

$$
K_{z}(x, y)=-C^{\top} \widetilde{K}_{z}(x, y) D
$$

where $C=[0, I]^{\top}, D=[I, 0]^{\top}$ and finally

$$
\widetilde{K}_{z}(x, y):= \begin{cases}\psi_{z}(x) \mathcal{P}_{z} \psi_{z}(y)^{-1} & 0 \leq x<y \leq 1 \\ \psi_{z}(x)\left(I-\mathcal{P}_{z}\right) \psi_{z}^{-1}(y) & 0 \leq y<x \leq 1\end{cases}
$$

for $\mathcal{P}_{z}:=\left[R_{0}+R_{1} \psi_{z}(1)\right]^{-1} R_{1} \psi_{z}(1)=R_{z}^{-1} R_{1} \psi_{z}(1)$.

REMARK 3.7. It is worth to note that $\widetilde{K}$ is not continuous on the diagonal whilst $K$ is. In fact, it is immediate to observe that the multiplication of $K$ by $C$ on the left and $D$ on the right, actually corresponds to consider the lower left block entry in the block decomposition of $\widetilde{K}_{z}$ (the identity is invisible in that block).

LEMMA 3.8. The form $\Theta_{z}$ is a trace class operator-valued one form.

Proof. Since the domain $\mathcal{D}$ of $\mathcal{A}_{z}$ is a dense $\left(z\right.$-independent) subspace of $\subset H^{2}\left([a, b], \mathbb{C}^{N}\right)$ and by taking into account Lemma 1.4, we get that the operator $\mathcal{A}_{z}^{-1}$ is a trace class operator on $L^{2}$. In fact $\mathcal{A}_{z}^{-1}$ is the composition of a bounded operator and of a trace class operator and hence it is in the trace class operator (being, in fact, trace class operators an ideal of the ring of all compact operators). Thus $\Theta_{z}$ is a trace class operator-valued one form. This concludes the proof.

The next proposition is crucial in order to establish the relation between the trace of $\Theta_{z}$ and the Brouwer degree of the determinant map.

Proposition 3.9. Under the above notation, we get

$$
\frac{1}{2 \pi i} \operatorname{Tr} \int_{\partial \Omega} \Theta_{z}=\operatorname{deg}(\rho, \Omega, 0)
$$

Proof. It is well-known that the trace of an integral operator belonging to the trace class can be computed by integrating the trace of its kernel. (Cfr. [LT98] for further details). Thus, by a direct calculation, we get:

$$
\operatorname{Tr} \Theta_{z}=\int_{0}^{1} \operatorname{Tr}\left[\mathrm{d} C_{z}(x) K_{z}(x, x)\right] \mathrm{d} x=-\int_{0}^{1} \operatorname{Tr}\left[J \mathrm{~d} B_{z}(x) \widetilde{K}_{z}(x, x)\right] \mathrm{d} x
$$

where the last equality follows by taking into account that

$$
\mathrm{d} C_{z}(x)=[I, 0] J \mathrm{~d} B_{z}(x)[0, I]^{\top} \quad \text { and } \quad K_{z}(x, y)=-C^{\top} \widetilde{K}_{z}(x, y) D
$$


as well as the commutativity of trace. On the other hand,

$$
\begin{aligned}
\operatorname{Tr}\left[J \mathrm{~d} B_{z}(x) \psi_{z}(x)\left(I-\mathcal{P}_{z}\right) \psi_{z}^{-1}(x)\right]= & \\
\operatorname{Tr}\left[J \mathrm{~d} B_{z}(x) \psi_{z}(x)\left(-\mathcal{P}_{z}\right) \psi_{z}^{-1}(x)\right]+ & \operatorname{Tr}\left[J \mathrm{~d} B_{z}(x)\right]= \\
& \operatorname{Tr}\left[J \mathrm{~d} B_{z}(x) \psi_{z}(x)\left(-\mathcal{P}_{z}\right) \psi_{z}^{-1}(x)\right] .
\end{aligned}
$$

Moreover

$$
\begin{aligned}
& \operatorname{Tr}\left[J \mathrm{~d} B_{z}(x) \psi_{z}(x)\left(-\mathcal{P}_{z}\right) \psi_{z}^{-1}(x)\right]= \\
& \quad-\operatorname{Tr}\left[J \mathrm{~d} B_{z}(x) \psi_{z}(x) \mathcal{P}_{z} \psi_{z}^{-1}(x)\right]=-\operatorname{Tr}\left[\mathrm{d} \psi_{z}^{\prime}(x) \mathcal{P}_{z} \psi_{z}^{-1}(x)-J B_{z}(x) \mathrm{d} \psi_{z}(x) \mathcal{P}_{z} \psi_{z}^{-1}(x)\right] \\
& =-\operatorname{Tr}\left[\mathrm{d} \psi_{z}^{\prime}(x) \mathcal{P}_{z} \psi_{z}^{-1}(x)-\psi_{z}^{\prime}(x) \psi_{z}^{-1}(x) \mathrm{d} \psi_{z}(x) \mathcal{P}_{z} \psi_{z}^{-1}(x)\right] \\
& =-\operatorname{Tr} \frac{\mathrm{d}}{\mathrm{d} x}\left[\mathrm{~d} \psi_{z}(x) \mathcal{P}_{z} \psi_{z}^{-1}(x)\right] .
\end{aligned}
$$

Putting together Equation (13) and Equation (14), we finally get

$$
\begin{aligned}
& \operatorname{Tr} \Theta_{z}=\int_{0}^{1} \operatorname{Tr}\left[\mathrm{d} C_{z}(x) K_{z}(x, x)\right] \mathrm{d} x \\
&=-\int_{0}^{1} \operatorname{Tr}\left[J \mathrm{~d} B_{z}(x) \widetilde{K}_{z}(x, x)\right] \mathrm{d} x \\
&=\int_{0}^{1} \frac{\mathrm{d}}{\mathrm{d} x} \operatorname{Tr}\left[\mathrm{d} \psi_{z}(x) \mathcal{P}_{z} \psi_{z}^{-1}(x)\right] \mathrm{d} x=\operatorname{Tr}\left[\mathrm{d} \psi_{z}(1) \mathcal{P}_{z} \psi_{z}^{-1}(1)\right] \\
&=\operatorname{Tr}\left[\mathrm{d} \psi_{z}(1) R_{z}^{-1} R_{1} \psi_{z}(1) \psi_{z}^{-1}(1)\right]=\operatorname{Tr}\left[\mathrm{d} \psi_{z}(1) R_{z}^{-1} R_{1}\right] .
\end{aligned}
$$

By Jacobi's formula, we get also that

$$
\mathrm{d} \log \operatorname{det} R_{z}=\operatorname{Tr}\left[R_{z}^{-1} \mathrm{~d} R_{z}\right]=\operatorname{Tr}\left[R_{z}^{-1} R_{1} \mathrm{~d} \psi_{z}(1)\right]=\operatorname{Tr}\left[\mathrm{d} \psi_{z}(1) R_{z}^{-1} R_{1}\right]
$$

and thus

$$
\operatorname{Tr} \Theta_{z}=\mathrm{d} \log \operatorname{det} R_{z} .
$$

Integrating over $\partial \Omega$, we than conclude that

$$
\frac{1}{2 \pi i} \int_{\partial \Omega} \Theta_{z}=\frac{1}{2 \pi i} \int_{\partial \Omega} \mathrm{d} \log \operatorname{det} R_{z}=\operatorname{deg}(\rho, \Omega, 0) .
$$

This concludes the proof.

REMARK 3.10. We observe that this computation is not affected if we replace the term $\psi_{z}(x)(I-$ $\left.\mathcal{P}_{z}\right) \psi_{z}^{-1}(y)$ entering in $\widetilde{K}_{z}(x, y)$ with $-\psi_{z}(x) \mathcal{P}_{z} \psi_{z}^{-1}(y)$.

Now we are in position to state and to prove the main result of this section. This result establish an equality between the spectral flow for a path of (unbouded) self-adjoint trace class resolvent operators and the degree index.

REMARK 3.11. Recall that if an operator has compact (trace class) resolvent, it is a Fredholm operator. The spectral flow is defined for a path of self-adjoint Fredholm operators, so it is also well defined for a path of self-adjoint operators having compact resolvent.

THEOREM 3.12. Under the above notation and if $\mathcal{A}$ is admissible, then we have

$$
\iota_{\mathrm{SP}}(\mathcal{A})=\iota_{\mathrm{PW}}(\psi, R) .
$$


Proof. We start to recall that pointwise, the path $\mathcal{A}$ is defined by $\mathcal{A}_{t}=\mathcal{A}_{0}+\mathcal{C}_{t}$.

First of all, without leading in generalities, we assume that $\left\{t \in(0,1) \mid \operatorname{ker} \mathcal{A}_{t} \neq\{0\}\right\}$ has has finite cardinality. If not, by [RS95, Theorem 4.22], for almost every $\delta \in \mathbb{R}, \mathcal{A}_{t}-\delta I$ has only regular crossings. (We refer to Section A and references therein, for the basic definitions and properties on the spectral flow). Since regular crossings are isolated, then on a compact interval are in a finite number. We observe also that

$$
\lim _{\delta \rightarrow 0} \frac{1}{2 \pi i} \operatorname{Tr} \int_{\partial \Omega} \mathrm{d} \mathcal{A}_{z}\left(\mathcal{A}_{z}-\delta I\right)^{-1}=\frac{1}{2 \pi i} \operatorname{Tr} \int_{\partial \Omega} \mathrm{d} \mathcal{A}_{z}\left(\mathcal{A}_{z}\right)^{-1},
$$

and by taking into account the homotopy invariance of the spectral flow, we get that if $\delta$ is sufficiently small then $\operatorname{sf}\left(\mathcal{A}_{t}, t \in[0,1]\right)=\operatorname{sf}\left(\mathcal{A}_{t}-\delta I, t \in[0,1]\right)$. So, we only need to prove the theorem in the case in which $\left\{t \in[0,1] \mid \operatorname{ker} \mathcal{A}_{t} \neq\{0\}\right\}=\left\{t_{1}, \ldots, t_{k}\right\}$. By using Lemma 3.3, we recall that

$$
\left\{\left(t_{1}, 0\right), \ldots,\left(t_{k}, 0\right)\right\}=\left\{z \in \Omega \mid \operatorname{ker} \mathcal{A}_{z} \neq\{0\}\right\} .
$$

By Lemma 2.4, for each $1 \leq i \leq k$, there is a rectangular neighborhood $\Omega_{i}$ of $t_{i}$ such that the decomposition $A_{z}=M_{z} N_{z}$ is well defined on $\Omega_{i}$. By Lemma $3.8 \mathrm{~d} \mathcal{A}_{z} \mathcal{A}_{z}^{-1}$ is a trace class operatorvalued one form and by Proposition 2.5 its trace is closed; thus we have

$$
\frac{1}{2 \pi i} \operatorname{Tr} \int_{\partial \Omega} \mathrm{d} \mathcal{A}_{z} \mathcal{A}_{z}^{-1}=\sum_{i=1}^{k} \frac{1}{2 \pi i} \operatorname{Tr} \int_{\partial \Omega_{i}} \mathrm{~d} \mathcal{A}_{z} \mathcal{A}_{z}^{-1}
$$

By invoking Proposition 2.7 we infer that

$$
\frac{1}{2 \pi i} \operatorname{Tr} \int_{\partial \Omega_{i}} \mathrm{~d} \mathcal{A}_{z} \mathcal{A}_{z}^{-1}=l-m
$$

where $l$ (resp. $m$ ) is the number of eigenvalues (counted with multiplicity) which cross the real axis with positive (resp. negative) derivative which is nothing but the spectral flow of the path $t \mapsto \mathcal{A}_{t}$ on a small neighborhood of $t_{i}$. So we have

$$
\sum_{i=1}^{k} \frac{1}{2 \pi i} \operatorname{Tr} \int_{\partial \Omega_{i}} \mathrm{~d} \mathcal{A}_{z} \mathcal{A}_{z}^{-1}=\operatorname{sf}\left(\mathcal{A}_{t}, t \in[0,1]\right)
$$

Thus we get

$$
\operatorname{sf}\left(\mathcal{A}_{t}, t \in[0,1]\right)=\frac{1}{2 \pi i} \operatorname{Tr} \int_{\partial \Omega} \Theta_{z} .
$$

By taking into account Definition 3.5, Remark 3.6 and Proposition 3.9, we get the thesis.

In many interesting applications often occurs that $P(x)$ (the principal symbol fo the MorseSturm operator) is actually represented by a positive definite (symmetric) matrix. In this case, in fact, the operator $\mathcal{A}_{t}$ has a well-defined Morse index for each $t \in[0,1]$. Denoting the Morse index of $\mathcal{A}_{t}$ by $m^{-}\left(\mathcal{A}_{t}\right)$, then we get the following immediate consequence.

Corollary 3.13. Under the assumption of Theorem 3.12 and assuming that $P(x)$ is positive definite for every $x \in[0,1]$, then we get

$$
m^{-}\left(\mathcal{A}_{0}\right)-m^{-}\left(\mathcal{A}_{1}\right)=\iota_{P W}(\psi, R) .
$$

Proof. The proof readily follows by Theorem 3.12 and Equation (22).

\subsection{Hill's determinant formula}

In this paragraph we establish the relation between the trace formula proved in Theorem 3.12 and the classical Hill's determinant formula. For, let us consider the eigenvalues problem for the standard Morse-Sturm system, given by

$$
-\frac{d}{d x}\left[P(x) \frac{d u}{d x}+Q(x) u\right]+Q^{\top}(x) \frac{d u}{d x}+\left(G(x)+t G_{1}(x)\right) u=0, \quad x \in[0,1]
$$


for $\lambda \in \mathbb{R}, Q \in \mathscr{C}^{1}([0,1], \operatorname{Mat}(N, \mathbb{R})), P, R, R_{1} \in \mathscr{C}^{1}([0,1], \operatorname{Sym}(N))$ and $P(x)$ is non-degenerate for every $x \in[0,1]$. By making use of the Legendre transformation, the linear system given in Equation (15) reduces to the following Hamiltonian system

$$
z^{\prime}(x)=J B_{t}(x) z(x)
$$

Let $\Lambda_{0}$ and $\Lambda_{1}$ be two Lagrangian subspaces of $\left(\mathbb{R}^{2 n}, \omega_{0}\right)$. Let $Z_{0}$ and $Z_{1}$ be two $2 n \times n$ matrix such that the spans of their column vectors are $\Lambda_{0}$ and $\Lambda_{1}$ respectively and we call them the Lagrangian frames of $\Lambda_{0}$ and $\Lambda_{1}$. Let us denote by $\psi_{\lambda}$ the fundamental solution of Equation (16).

It is well-known that the operator Morse-Sturm operator

$$
\mathcal{A}:=-\frac{d}{d x}\left[P(x) \frac{d}{d x}+Q(x)\right]+Q^{\top}(x) \frac{d}{d x}+G(x)
$$

is self-adjoint in $L^{2}\left(I, \mathbb{R}^{n}\right)$ with dense domain

$$
\mathcal{D}\left(\Lambda_{0}, \Lambda_{1}\right)=\left\{u \in H^{2}\left([0,1], \mathbb{R}^{n}\right) \mid z(0) \in \Lambda_{0}, z(1) \in \Lambda_{1}\right\} .
$$

We assume $\mathcal{A}$ is non-degenerate (meaning that $\operatorname{ker} \mathcal{A}=\{0\}$ ). It is clear that $\lambda$ is a non-zero eigenvalue of $\mathcal{A}$ if and only if $-\frac{1}{\lambda}$ is an eigenvalue of $\mathcal{G}_{1} \mathcal{A}^{-1}$, where $\mathcal{G}_{1}$ is the operator on $L^{2}$ pointwise induced by $G_{1}$. The following result holds. See [HW16, Theorem 1.1])

TheOrem 3.14. (Hu $\&$ Wang, 2016) If $\mathcal{A}$ is non-degenerate, then we have

$$
\prod_{j}\left(1-\lambda_{j}^{-1}\right)=\operatorname{det}\left(\psi_{1}(1) Z_{0}, Z_{1}\right) \cdot \operatorname{det}\left(\psi_{0}(1) Z_{0}, Z_{1}\right)^{-1}
$$

where the left-hand side is an infinite product which runs on all the eigenvalues $\lambda_{j}$ counted with multiplicity.

We observe that the left-hand side in Equation (17) is the Fredholm determinant of $\operatorname{det}(I+$ $\left.\mathcal{G}_{1} \mathcal{A}^{-1}\right)$. Since $Z_{0}$ is a basis of $V_{0}$, we have $Z_{0}^{*} J Z_{0}=0$. Similarly we have $Z_{1}^{*} J Z_{1}=0$. Then $\left(\begin{array}{c}\left(J Z_{0}\right)^{*} \\ 0\end{array}\right)$ and $\left(\begin{array}{c}0 \\ \left(J Z_{1}\right)^{*}\end{array}\right)$ are $R_{0}$ and $R_{1}$ of the boundary condition $z_{0} \in \Lambda_{0}, z_{1} \in \Lambda_{1}$. Choose matrix $P, Q$ such that $\left(\begin{array}{cc}R_{0} & R_{1} \\ P & Q\end{array}\right)$ is invertible.

Then we have $\left(\begin{array}{cc}R_{0} & R_{1} \\ P & Q\end{array}\right)\left(\begin{array}{cc}-Z_{0} & 0 \\ 0 & Z_{1}\end{array}\right)=\left(\begin{array}{cc}0 & 0 \\ -P Z_{0} & Q Z_{0}\end{array}\right)$. Then the rank of $\left(\begin{array}{cc}0 & 0 \\ -P Z_{0} & Q Z_{0}\end{array}\right)$

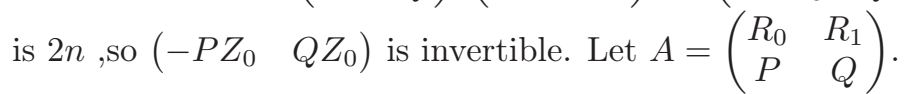

Note that

$$
\begin{aligned}
\operatorname{det}\left(\psi Z_{0}, Z_{1}\right) \operatorname{det} A & =\operatorname{det}\left(\left(\begin{array}{cc}
R_{0} & R_{1} \\
P & Q
\end{array}\right)\left(\begin{array}{ccc}
I d & -Z_{0} & 0 \\
\psi & 0 & Z_{1}
\end{array}\right)\right) \\
& =\operatorname{det}\left(\begin{array}{ccc}
R_{0}+R_{1} \psi & 0 & 0 \\
P+Q \psi & -P Z_{0} & Q Z_{1}
\end{array}\right)=\operatorname{det}\left(R_{0}+R_{1} \psi\right) \operatorname{det}\left(\left(\begin{array}{lll}
-P Z_{0} & \left.\left.Q Z_{1}\right)\right)
\end{array}\right.\right.
\end{aligned}
$$

It follows that $\rho(z)=\operatorname{det}\left(\psi_{z}(1)\right) \operatorname{det}(A) \operatorname{det}\left(\left(\begin{array}{lll}-P Z_{0} & Q Z_{1}\end{array}\right)\right)^{-1}$. Then we have

$$
\prod_{j}\left(1-\lambda_{j}^{-1}\right)=\rho(1) \rho(0)^{-1}
$$

REMARK 3.15. In fact, in the proof of 3.14, they proved that

$$
\operatorname{det}\left(1+z \mathcal{G}_{1} \mathcal{A}^{-1}\right)=\rho(z) \rho(0)^{-1}, z \in \mathbb{C} .
$$

Corollary 3.16. Let $f(z)=\operatorname{det}\left(1+\left(t \mathcal{G}_{1}+i s I\right) \mathcal{A}^{-1}\right)$. Assume that $\mathcal{A}$ and $\mathcal{A}+\mathcal{G}_{1}$ are both invertible. Let $\Omega=[0,1] \times[-1,1]$. We have $\operatorname{deg}(f, \Omega, 0)=\operatorname{sf}\left(\mathcal{A}+t \mathcal{G}_{1}, t \in[0,1]\right)$. 
Proof. By Proposition 3.9 and Theorem 3.12, we only need to show that $f(z)=\rho(z) \rho(0)^{-1}$. Note that if $\mathcal{A}+\mathcal{G}_{1}$ is invertible, we have

$$
1+\left(t \mathcal{G}_{1}+i s I\right) \mathcal{A}^{-1}=\left(1+i s I\left(\mathcal{A}+t \mathcal{G}_{1}\right)^{-1}\right)\left(1+t \mathcal{G}_{1} \mathcal{A}^{-1}\right) .
$$

With the same perturbation method in theorem 3.12 , we can assume that $\left(A+t G_{1}\right)$ is invertible except for a finite number of $t$. By the multiplication property of Fredholm determinant and remark 3.15 , we have $f(z)=\rho(z) \rho(t)^{-1} \rho(t) \rho(0)^{-1}=\rho(z) \rho(0)^{-1}$ on a dense subset of $\Omega$. Then the equation holds for all $z \in \Omega$ by the continuity of Fredholm determinant.

\section{Parity of the degree index and instability of periodic orbits}

In this section, by using Theorem 3.12, a Morse-type index theorem together with a characterization of the linear instability for a periodic orbit of a Hamiltonian system, we establish a sufficient condition in terms of the degree index for detecting the linear instability.

Let $T \mathbb{R}^{n} \cong \mathbb{R}^{n} \oplus \mathbb{R}^{n}$ be the tangent space of $\mathbb{R}^{n}$ endowed with coordinates $(q, v)$. Given $T>0$ and the Lagrangian function $L \in \mathscr{C}^{2}\left([0, T] \times T \mathbb{R}^{n}, \mathbb{R}\right)$, we assume that the following two assumptions hold

(L1) $L$ is non-degenerate with respect to $v$, meaning that the quadratic form

$$
\left\langle\nabla_{v v} L(t, q, v) w, w\right\rangle \quad \text { is non-degenerate } \quad \forall t \in[0, T], \quad \forall(q, v) \in T \mathbb{R}^{n}
$$

(L2) $L$ is exactly quadratic in the velocities $v$ meaning that the function $L(t, q, v)$ is a polynomial of degree at most 2 with respect to $v$.

Under the assumption (L1) the Legendre transform

$$
\mathscr{L}_{L}:[0, T] \times T \mathbb{R}^{n} \rightarrow[0, T] \times T^{*} \mathbb{R}^{n}, \quad(t, q, v) \mapsto\left(t, q, D_{v} L(t, q, v)\right)
$$

is a $\mathscr{C}^{1}$ (local) diffeomorphism. The Fenchel transform of $L$ is the non autonomous Hamiltonian on $T^{*} \mathbb{R}^{n}$

$$
H(t, q, p):=\max _{v \in T_{q} M}(p[v]-L(t, q, v))=p[v(t, q, p)]-L(t, q, v(t, q, p))
$$

where $(t, q, v(t, q, p))=\mathscr{L}_{L}^{-1}(t, q, p)$.

REMARK 4.1. The assumption (L2) is in order to guarantee that the action functional is twice Frechét differentiable. It is well-known, in fact, that the smoothness assumption on the Lagrangian is in general not enough. The growth condition required in (L2) is related to the regularity of the Nemitski operators. For further details we refer to [PWY19] and references therein.

We denote by $H:=W^{1,2}\left([0, T], \mathbb{R}^{n}\right)$ be the space of paths having Sobolev regularity $W^{1,2}$ and we define the Lagrangian action functional $\mathbb{A}: H \rightarrow \mathbb{R}$ as follows

$$
A(x)=\int_{0}^{T} L\left(t, x(t), x^{\prime}(t)\right) d t .
$$

Let $Z \subset \mathbb{R}^{n} \oplus \mathbb{R}^{n}$ be a linear subspace and let us consider the linear subspace

$$
H_{Z}:=\{x \in H \mid(x(0), x(T)) \in Z\} .
$$

Notation 4.2. In what follows we shall denote by $A_{Z}$ the restriction of the action $A$ onto $H_{Z}$; thus in symbols we have $A_{Z}:=\left.A\right|_{H_{Z}}$.

It is well-know that critical point of the functional $A$ on the $H_{Z}$ are weak (in the Sobolev sense) solutions of the following boundary value problem

$$
\left\{\begin{array}{l}
\frac{d}{d t} \partial_{v} L\left(t, x(t), x^{\prime}(t)\right)=\partial_{q} L\left(t, x(t), x^{\prime}(t)\right), \quad t \in[0, T] \\
(x(0), x(T)) \in Z, \quad\left(\partial_{v} L\left(0, x(0), x^{\prime}(0)\right),-\partial_{v} L\left(T, x(T), x^{\prime}(T)\right)\right) \in Z^{\perp}
\end{array}\right.
$$

where $Z^{\perp}$ denotes the orthogonal complement of $Z$ in $T^{*} \mathbb{R}^{n}$ and up to standard elliptic regularity arguments, classical (i.e. smooth) solutions. 
REMARK 4.3. We observe, in fact, that there is an identification of $Z \times Z^{\perp}$ and the conormal subspace of $Z$, namely $N^{*}(Z)$ in $T^{*} \mathbb{R}^{n}$. For further details, we refer the interested reader to [APS08].

We assume that $x \in H_{Z}$ is a classical solution of the boundary value problem given in Equation (18). We observe that, by assumption (L2) the functional $A$ is twice Fréchet differentiable on $H$. Being the evaluation map from $H$ onto $H_{Z}$ a smooth submersion (cfr. [Kli83] for further details), also the restriction $A_{Z}$ is twice Fréchet differentiable and by this we get that $d^{2} A_{Z}(x)$ coincides with $D^{2} A_{Z}(x)$.

By computing the second variation of $A_{Z}$ at $x$, we get the index form $I$ defined by

$$
\begin{gathered}
d^{2} A_{Z}(x)[\xi, \eta]:=I[\xi, \eta]=\int_{0}^{T}\left[\left\langle P(t) \xi^{\prime}+Q(t) \xi, \eta^{\prime}\right\rangle+\left\langle Q^{\top}(t) \xi^{\prime}, \eta\right\rangle+\langle R(t) \xi, \eta\rangle\right] d t, \quad \forall \xi, \eta \in H_{Z} \\
\text { where } P(t):=\partial_{v v} L\left(t, x(t), x^{\prime}(t)\right), \quad Q(t):=\partial_{q v} L\left(t, x(t), x^{\prime}(t)\right) \\
\quad \text { and finally } R(t):=\partial_{q q} L\left(t, x(t), x^{\prime}(t)\right) .
\end{gathered}
$$

Now, by linearizing the ODE given in Equation (18) at $x$, we finally get the (linear) Morse-Sturm boundary value problem defined as follows

$$
\left\{\begin{array}{l}
-\frac{d}{d t}\left[P(t) u^{\prime}+Q(t) u\right]+Q^{\top}(t) u^{\prime}+R(t) u=0, \quad t \in[0, T] \\
(u(0), u(T)) \in Z, \quad\left(P u^{\prime}(0)+Q(0) u(0),-\left[P(T) u^{\prime}(T)+Q(T) u(T)\right]\right) \in Z^{\perp}
\end{array}\right.
$$

We observe that $u$ is a weak (in the Sobolev sense) solution of the boundary value problem given in Equation (19) if and only if $u \in \operatorname{ker} I$. Moreover, by elliptic bootstrap it follows that $u$ is a smooth solution.

Let us now consider the standard symplectic space $T^{*} \mathbb{R}^{n} \cong \mathbb{R}^{n} \oplus \mathbb{R}^{n}$ endowed with the canonical symplectic form

$$
\omega_{0}\left(\left(p_{1}, q_{1}\right),\left(p_{2}, q_{2}\right)\right):=\left\langle p_{1}, q_{2}\right\rangle-\left\langle q_{1}, p_{2}\right\rangle
$$

Denoting by $J_{0}$ the (standard) complex structure namely the automorphism $J_{0}: T^{*} \mathbb{R}^{n} \rightarrow T^{*} \mathbb{R}^{n}$ defined by $J_{0}(p, q)=(-q, p)$ and whose associated matrix is given by

$$
J_{0}=\left(\begin{array}{cc}
0 & -I \\
I & 0
\end{array}\right)
$$

it immediately follows that $\omega_{0}\left(z_{1}, z_{2}\right):=\left\langle J_{0} z_{1}, z_{2}\right\rangle$ for all $z_{1}, z_{2} \in T^{*} \mathbb{R}^{n}$.

Notation 4.4. In what follows, $T^{*} \mathbb{R}^{n}$ is endowed with a coordinate system $z=(p, q)$, where $p=\left(p_{1}, \ldots, p_{n}\right) \in \mathbb{R}^{n}$ and $q=\left(q_{1}, \ldots, q_{n}\right) \in \mathbb{R}^{n}$. we shall refer to $q$ as configuration variables and to $p$ as the momentum variables.

By setting $z(t):=\left(P(t) u^{\prime}(t)+Q(t) u(t), u(t)\right)^{\top}$, the Morse-Sturm equation reduces to the following (first order) Hamiltonian system in the standard symplectic space

$$
\begin{aligned}
& z^{\prime}(t)=J_{0} B(t) z(t), \quad t \in[0, T] \quad \text { where } \\
& \qquad B(t):=\left[\begin{array}{cc}
P^{-1}(t) & -P^{-1}(t) Q(t) \\
-Q^{\top}(t) P^{-1}(t) & Q^{\top}(t) P^{-1}(t) Q(t)-R(t)
\end{array}\right]
\end{aligned}
$$

We now define the double standard symplectic space $\left(\mathbb{R}^{2 n} \oplus \mathbb{R}^{2 n},-\omega_{0} \oplus \omega_{0}\right)$ and we introduce the matrix $\widetilde{J}_{0}:=\operatorname{diag}\left(-J_{0}, J_{0}\right)$ where $\operatorname{diag}(*, *)$ denotes the $2 \times 2$ diagonal block matrix. In this way, the subspace $L_{Z}$ given by

$$
L_{Z}:=\widetilde{J}_{0}\left(Z^{\perp} \oplus Z\right)
$$

is thus Lagrangian. 
Notation 4.5. The following notation will be used throughout the paper. If $x$ is a solution of (18) we denote by $z_{x}$ the corresponding function defined by

$$
\left(t, z_{x}(t)\right)=\mathscr{L}_{L}\left(t, x(t), x^{\prime}(t)\right)
$$

Let us now consider the path $s \mapsto \mathcal{L}_{s}$ of unbounded Hamiltonian operators that are self-adjoint in $L^{2}$ and defined on the domain $D(T, L)$ :

$$
\mathcal{L}_{s}:=-J_{0} \frac{d}{d t}-B_{s}(t)
$$

where $s \mapsto B_{s}(t)$ is a $\mathscr{C}^{1}$ path of symmetric matrices such that $B_{0}(t)=0_{2 n}$ and $B_{1}(t)=B(t)$.

LEMMA 4.6. (Spectral flow formula) Under the above notation, the following equality holds

$$
-\operatorname{sf}\left(\mathcal{L}_{s}, s \in[0,1]\right)=\iota^{\mathrm{CLM}}(L, \operatorname{Gr} \psi(t), t \in[0, T])
$$

where $\psi$ denotes the solution of

$$
\left\{\begin{array}{l}
\frac{d}{d t} \psi(t)=J_{0} B(t) \psi(t), \quad t \in[0, T] \\
\psi(0)=I_{2 n} .
\end{array}\right.
$$

Proof. For the proof of this result, we refer the interested reader to [HS09, Theorem 2.5, Equation (2.7) \& Equation (2.19)].

REMARK 4.7. The basic idea behind the proof of Proposition 4.6 is to perturb the path $s \mapsto \mathcal{L}_{s}$ in order to get regular crossing and without changing the spectral flow (as consequence of the fixed endpoints homotopy invariance). Once this has been done, for concluding, it is enough to prove that the local contribution at each crossing instant to the spectral flow is the opposite of the local contribution to the Maslov index. This can be achieved by comparing the crossing forms as in [HS09, Lemma 2.4] and to prove that the crossing instants for the path $s \mapsto \mathcal{L}_{s}$ are the same as the crossing instants of the path $s \mapsto \operatorname{Gr} \psi_{s}$ and at each crossing $s_{0}$ the kernel dimension of the operator $\mathcal{L}_{s_{0}}$ is equal to the $\operatorname{dim}\left(L \cap \operatorname{Gr} \psi_{s_{0}}\right)$. The conclusion follows once again by using the homotopy properties of the $\iota^{\mathrm{CLM}}$-index and the spectral flow.

Definition 4.8. Let $x$ be a critical point of $A$. We denote by $\iota_{Z}(x)$ the spectral index of $x$ namely

$$
\iota_{Z}(x):=-\operatorname{sf}\left(\mathcal{L}_{s}, s \in[0,1]\right) .
$$

Let $z_{x}$ be defined in Equation (21). We define the Maslov index of $z_{x}$ as the integer given by

$$
\iota_{L_{Z}}\left(z_{x}\right):=\iota^{\mathrm{CLM}}\left(L_{Z}, \operatorname{Gr} \psi(t), t \in[0, T]\right)
$$

where $\psi$ denotes the fundamental solution of the Hamiltonian system given in Equation (20).

Proposition 4.9. Under the previous notation, if $x$ is a critical point of $A_{Z}$, then $\iota_{Z}(x)$ is welldefined. Moreover the following equality holds

$$
\iota_{Z}(x)=\iota_{L_{Z}}\left(z_{x}\right)
$$

Proof. For the proof of this result we refer the reader to [HS09, Theorem 2.5].

We close this section with the following characterization of the linear instability of a periodic orbit through the parity of the degree index.

THEOREM 4.10. Let $x$ be a T-periodic solution of Equation (18). If one of the following two alternatives hold

$$
\begin{aligned}
& (\mathbf{O R}) x \text { is orientation preserving and } \iota_{\mathrm{PW}}(x)+n \text { is odd } \\
& (\mathbf{N O R}) x \text { is non orientation preserving and } \iota_{\mathrm{PW}}(x)+n \text { is even }
\end{aligned}
$$

then $x$ is linearly unstable. 
Proof. We prove the theorem in the case (OR) being the other completely analogous. Let $x$ be a $T$-periodic orientation preserving solutions and let $z_{x}$ be the solution of the corresponding Hamiltonian system defined in Equation (21).

By Proposition 4.9, we get that

$$
\iota^{\mathrm{CLM}}(\Delta, \operatorname{Gr} \psi(t), t \in[0, T])=-\operatorname{sf}\left(\mathcal{L}_{s}, s \in[0,1]\right) .
$$

By [HS09, Equation (3.18)], we get that $\operatorname{sf}\left(\mathcal{L}_{s}, s \in[0,1]\right)=\operatorname{sf}\left(\mathcal{A}_{s}, s \in[0,1]\right)$ where $s \mapsto \mathcal{A}_{s}$ is the path of second order self-adjoint Fredholm operators on $L^{2}$ with dense domain

$$
E^{2}\left([0, T], \mathbb{R}^{n}\right)=\left\{u \in H^{2}\left([0, T], \mathbb{R}^{n}\right) \mid u(0)=u(T) \text { and } u^{\prime}(0)=u^{\prime}(T)\right\} .
$$

By invoking Theorem 3.12, then we get

$$
\begin{aligned}
\iota^{\mathrm{CLM}}(\Delta, \operatorname{Gr} \psi(t), t \in[0, T])+n=-\operatorname{sf}\left(\mathcal{L}_{s}, s \in[0,1]\right) & +n \\
& =-\operatorname{sf}\left(\mathcal{A}_{s}, s \in[0,1]\right)+n=-\iota_{\mathrm{PW}}(\psi, R)+n .
\end{aligned}
$$

Now since the parity of $-\iota_{\mathrm{PW}}(\psi, R)+n$ and $\iota_{\mathrm{PW}}(\psi, R)+n$ both coincides, the result directly follows by [PWY19, Theorem 1]. This concludes the proof.

\section{A A recap on the Maslov index and spectral flow}

In this section, we provide the basic definition and properties on the Maslov index and the spectral flow. Our basic references are [BJP14a, BJP14b, KOP19, PWY19] and references therein.

\section{A.1 On the Maslov index}

Given a $2 n$-dimensional (real) symplectic space $(V, \omega)$, a Lagrangian subspace of $V$ is an $n$-dimensional subspace $L \subset V$ such that $L=L^{\omega}$ where $L^{\omega}$ denotes the symplectic orthogonal, i.e. the orthogonal with respect to the symplectic structure. We denote by $\Lambda=\Lambda(V, \omega)$ the Lagrangian Grassmannian of $(V, \omega)$, namely the set of all Lagrangian subspaces of $(V, \omega)$

$$
\Lambda(V, \omega):=\left\{L \subset V \mid L=L^{\omega}\right\} .
$$

It is well-known that $\Lambda(V, \omega)$ is a manifold. For each $L_{0} \in \Lambda$, let

$$
\Lambda^{k}\left(L_{0}\right):=\left\{L \in \Lambda(V, \omega) \mid \operatorname{dim}\left(L \cap L_{0}\right)=k\right\} \quad k=0, \ldots, n .
$$

Each $\Lambda^{k}\left(L_{0}\right)$ is a real compact, connected submanifold of codimension $k(k+1) / 2$. The topological closure of $\Lambda^{1}\left(L_{0}\right)$ is the Maslov cycle that can be also described as follows

$$
\Sigma\left(L_{0}\right):=\bigcup_{k=1}^{n} \Lambda^{k}\left(L_{0}\right)
$$

The top-stratum $\Lambda^{1}\left(L_{0}\right)$ is co-oriented meaning that it has a transverse orientation. To be more precise, for each $L \in \Lambda^{1}\left(L_{0}\right)$, the path of Lagrangian subspaces $(-\delta, \delta) \mapsto e^{t J} L \operatorname{cross} \Lambda^{1}\left(L_{0}\right)$ transversally, and as $t$ increases the path points to the transverse direction. Thus the Maslov cycle is two-sidedly embedded in $\Lambda(V, \omega)$. Based on the topological properties of the Lagrangian Grassmannian manifold, it is possible to define a fixed endpoints homotopy invariant called Maslov index.

Definition A.1. Let $L_{0} \in \Lambda(V, \omega)$ and let $\ell:[0,1] \rightarrow \Lambda(V, \omega)$ be a continuous path. We define the Maslov index $\iota^{\mathrm{CLM}}$ as follows:

$$
\iota^{\mathrm{CLM}}\left(L_{0}, \ell(t) ; t \in[a, b]\right):=\left[e^{-\varepsilon J} \ell(t): \Sigma\left(L_{0}\right)\right]
$$

where the right hand-side denotes the intersection number and $0<\varepsilon<<1$. 
For further reference we refer the interested reader to [CLM94] and references therein.

REMARK A.2. It is worth noticing that for $\varepsilon>0$ small enough, the Lagrangian subspaces $e^{-\varepsilon J} \ell(a)$ and $e^{-\varepsilon J} \ell(b)$ are off the singular cycle.

One efficient way to compute the Maslov index, was introduced by authors in [RS93] via crossing forms. Let $\ell$ be a $\mathscr{C}^{1}$-curve of Lagrangian subspaces such that $\ell(0)=L$ and let $W$ be a fixed Lagrangian subspace transversal to $L$. For $v \in L$ and small enough $t$, let $w(t) \in W$ be such that $v+w(t) \in \ell(t)$. Then the form

$$
Q(v)=\left.\frac{d}{d t}\right|_{t=0} \omega(v, w(t))
$$

is independent on the choice of $W$. A crossing instant for $\ell$ is an instant $t \in[a, b]$ such that $\ell(t)$ intersects $W$ nontrivially. At each crossing instant, we define the crossing form as

$$
\Gamma(\ell(t), W, t)=\left.Q\right|_{\ell(t) \cap W} .
$$

A crossing is termed regular if the crossing form is non-degenerate. If $\ell$ is regular meaning that it has only regular crossings, then the Maslov index is equal to

$$
\iota^{\mathrm{CLM}}(W, \ell(t) ; t \in[a, b])=\mathrm{n}_{+}(\Gamma(\ell(a), W ; a))+\sum_{a<t<b} \operatorname{sgn}\left(\Gamma(\ell(t), W ; t)-\iota_{\mathrm{MOR}}(\Gamma(\ell(b), W ; b)\right.
$$

where the summation runs over all crossings $t \in(a, b)$ and $\mathrm{n}_{+}, \iota_{\mathrm{MOR}}$ are the dimensions of the positive and negative spectral spaces, respectively and $\operatorname{sgn}:=\mathrm{n}_{+}-\iota_{\mathrm{MOR}}$ is the signature. (We refer the interested reader to [LZ00] and [HS09, Equation (2.15)]). We close this section by recalling some useful properties of the Maslov index.

Property I (Reparametrization invariance). Let $\psi:[a, b] \rightarrow[c, d]$ be a continuous and piecewise smooth function with $\psi(a)=c$ and $\psi(b)=d$, then

$$
\iota^{\mathrm{CLM}}(W, \ell(t))=\iota^{\mathrm{CLM}}(W, \ell(\psi(t))) .
$$

Property II (Homotopy invariance with respect to the ends). For any $s \in[0,1]$, let $s \mapsto \ell(s, \cdot)$ be a continuous family of Lagrangian paths parametrised on $[a, b]$ and such that $\operatorname{dim}(\ell(s, a) \cap W)$ and $\operatorname{dim}(\ell(s, b) \cap W)$ are constants, then

$$
\iota^{\mathrm{CLM}}(W, \ell(0, t) ; t \in[a, b])=\iota^{\mathrm{CLM}}(W, \ell(1, t) ; t \in[a, b]) .
$$

Property III (Path additivity). If $a<c<b$, then

$$
\iota^{\mathrm{CLM}}(W, \ell(t) ; t \in[a, b])=\iota^{\mathrm{CLM}}(W, \ell(t) ; t \in[a, c])+\iota^{\mathrm{CLM}}(W, \ell(t) ; t \in[c, b])
$$

Property IV (Symplectic invariance). Let $\Phi:[a, b] \rightarrow \operatorname{Sp}(2 n, \mathbb{R})$. Then

$$
\iota^{\mathrm{CLM}}(W, \ell(t) ; t \in[a, b])=\iota^{\mathrm{CLM}}(\Phi(t) W, \Phi(t) \ell(t) ; t \in[a, b]) .
$$

In the standard symplectic space $\left(\mathbb{R}^{2 n}, \omega\right)$ we denote by $J$ the standard symplectic matrix defined by $J=\left[\begin{array}{cc}0 & -I \\ I & 0\end{array}\right]$. The symplectic form $\omega$ can be represented with respect to the Euclidean product $\langle\cdot, \cdot\rangle$ by $J$ as follows $\omega\left(z_{1}, z_{2}\right)=\left\langle J z_{1}, z_{2}\right\rangle$ for every $z_{1}, z_{2} \in \mathbb{R}^{2 n}$. We consider the 1-codimensional (algebraic) subvariety

$$
\operatorname{Sp}(2 n, \mathbb{R})^{0}:=\{M \in \operatorname{Sp}(2 n, \mathbb{R}) \mid \operatorname{det}(M-I)=0\} \subset \operatorname{Sp}(2 n, \mathbb{R})
$$

and let us define

$$
\operatorname{Sp}(2 n, \mathbb{R})^{*}=\operatorname{Sp}(2 n, \mathbb{R}) \backslash \operatorname{Sp}(2 n, \mathbb{R})^{0}=\operatorname{Sp}(2 n, \mathbb{R})^{+} \cup \operatorname{Sp}(2 n, \mathbb{R})^{-}
$$


where

$$
\begin{array}{r}
\operatorname{Sp}(2 n, \mathbb{R})^{+}:=\{M \in \operatorname{Sp}(2 n, \mathbb{R}) \mid \operatorname{det}(M-I)>0\} \quad \text { and } \\
\qquad \operatorname{Sp}(2 n, \mathbb{R})^{-}:=\{M \in \operatorname{Sp}(2 n, \mathbb{R}) \mid \operatorname{det}(M-I)<0\} .
\end{array}
$$

For any $M \in \operatorname{Sp}(2 n, \mathbb{R})^{0}, \operatorname{Sp}(2 n, \mathbb{R})^{0}$ is co-oriented at the point $M$ by choosing as positive direction the direction determined by $\left.\frac{d}{d t} M e^{t J}\right|_{t=0}$ with $t \geq 0$ sufficiently small. We recall that $\operatorname{Sp}(2 n, \mathbb{R})^{+}$and $\operatorname{Sp}(2 n, \mathbb{R})^{-}$are two path connected components of $\operatorname{Sp}(2 n, \mathbb{R})^{*}$ which are simple connected in $\operatorname{Sp}(2 n, \mathbb{R})$. (For the proof of these facts we refer, for instance, the interested reader to [Lon02, pag.58-59] and references therein). Following authors in [LZ00, Definition 2.1] we start by recalling the following definition.

Definition A.3. Let $\psi:[a, b] \rightarrow \operatorname{Sp}(2 n, \mathbb{R})$ be a continuous path. Then there exists an $\varepsilon>0$ such that for every $\theta \in[-\varepsilon, \varepsilon] \backslash\{0\}$, the matrices $\psi(a) e^{J \theta}$ and $\psi(b) e^{J \theta}$ lying both out of $\operatorname{Sp}(2 n, \mathbb{R})^{0}$. We define the $\iota_{1}$-index or the Maslov-type index as follows

$$
\iota_{1}(\psi):=\left[e^{-J \varepsilon} \psi: \operatorname{Sp}(2 n, \mathbb{R})^{0}\right]
$$

where the (RHS) denotes the intersection number between the perturbed path $t \mapsto e^{-J \varepsilon} \psi(t)$ with the singular cycle $\operatorname{Sp}(2 n, \mathbb{R})^{0}$.

Through the parity of the $\iota_{1}$-index it is possible to locate endpoints of the perturbed symplectic path $t \mapsto e^{-J \varepsilon} \psi(t)$.

Lemma A.4. ([Lon02, Lemma 5.2.6]) Let $\psi:[a, b] \rightarrow \operatorname{Sp}(2 n, \mathbb{R})$ be a continuous path. The following characterization holds

$\iota_{1}(\psi)$ is even $\Longleftrightarrow$ both the endpoints $e^{-\varepsilon J} \psi(a)$ and $e^{-\varepsilon J} \psi(b)$ lie in $\operatorname{Sp}(2 n, \mathbb{R})^{+}$or in $\operatorname{Sp}(2 n, \mathbb{R})^{-}$.

We close this section with a rather technical result which will be used in the proof of the main instability criterion.

Lemma A.5. ([HS10, Lemma 3.2]. ) Let $\psi:[a, b] \rightarrow \operatorname{Sp}(2 n, \mathbb{R})$ be a continuous symplectic path such that $\psi(0)$ is linearly stable.

1. If $1 \notin \sigma(\psi(a))$ then there exists $\varepsilon>0$ sufficiently small such that $\psi(s) \in \operatorname{Sp}(2 n, \mathbb{R})^{+}$for $|s| \in(0, \varepsilon)$.

2. We assume that $\operatorname{dim} \operatorname{ker}(\psi(a)-I)=m$ and $\left.\psi(a)^{\top} J \psi^{\prime}(a)\right|_{V}$ is non-singular for $V:=\psi^{-1}(a) \mathbb{R}^{2 m}$. If ind $\left(\left.\psi(a)^{\top} J \psi^{\prime}(a)\right|_{V}\right)$ is even [resp. odd] then there exists $\delta>0$ sufficiently small such that $\psi(s) \in \operatorname{Sp}(2 n, \mathbb{R})^{+}$resp. $\left.\psi(s) \in \operatorname{Sp}(2 n, \mathbb{R})^{-}\right]$for $|s| \in(0, \delta)$.

REMARK A.6. Knowing that $M \in \operatorname{Sp}^{0}(2 n, \mathbb{R})$, without any further information, it is not possible a priori to locate in which path connected components of $\operatorname{Sp}(2 n, \mathbb{R})^{*}$ is located the perturbed matrix $e^{ \pm \delta J} M$ for arbitrarily small positive $\delta$. However if $M$ is linearly stable, we get the following result.

LemmA A.7. Let $M \in \operatorname{Sp}(2 n, \mathbb{R})$ be a linearly stable symplectic matrix (meaning that $\mathfrak{s} M \subset \mathbb{U}$ and $M$ is diagonalizable). Then, there exists $\delta>0$ sufficiently small such that $e^{ \pm \delta J} M \in \operatorname{Sp}(2 n, \mathbb{R})^{+}$.

Proof. Let us consider the symplectic path pointwise defined by $M(\theta):=e^{-\theta J} M$. By a direct computation we get that

$$
\left.M(\theta)^{\top} J \frac{d}{d \theta} M(\theta)\right|_{\theta=0}=M^{\top} M
$$

We observe that $M^{\top} M$ is symmetric and positive semi-definite; moreover since $M$ invertible it follows that $M^{\top} M$ is actually positive definite. Thus, in particular, $\mathrm{n}_{-}\left(M^{\top} M\right)=0$. By invoking Lemma A.5 it follows that there exists $\delta>0$ such that $M( \pm \delta) \in \operatorname{Sp}(2 n, \mathbb{R})^{+}$. This concludes the proof. 


\section{A.2 On the spectral flow}

The aim of this subesection is to briefly recall the Definition and the main properties of the spectral flow for a continuous path of closed self-adjoint Fredholm operators $\mathcal{C} \mathcal{F}^{s a}(\mathcal{H})$ on the Hilbert space $H$. Our basic reference is [HPY19] and references therein.

Let $\mathcal{H}$ be a separable complex Hilbert space and let $A: \mathcal{D}(A) \subset \mathcal{H} \rightarrow \mathcal{H}$ be a self-adjoint Fredholm operator. By the Spectral decomposition Theorem (cf., for instance, [Kat80, Chapter III, Theorem 6.17]), there is an orthogonal decomposition $\mathcal{H}=E_{-}(A) \oplus E_{0}(A) \oplus E_{+}(A)$, that reduces the operator $A$ and has the property that

$$
\sigma(A) \cap(-\infty, 0)=\sigma\left(A_{E_{-}(A)}\right), \quad \sigma(A) \cap\{0\}=\sigma\left(A_{E_{0}(A)}\right), \quad \sigma(A) \cap(0,+\infty)=\sigma\left(A_{E_{+}(A)}\right) .
$$

Definition A.8. Let $A \in \mathcal{C F}^{s a}(\mathcal{H})$. We term $A$ essentially positive if $\sigma_{\text {ess }}(A) \subset(0,+\infty)$, essentially negative if $\sigma_{\text {ess }}(A) \subset(-\infty, 0)$ and finally strongly indefinite respectively if $\sigma_{\text {ess }}(A) \cap(-\infty, 0) \neq$ $\emptyset$ and $\sigma_{\text {ess }}(A) \cap(0,+\infty) \neq \emptyset$.

If $\operatorname{dim} E_{-}(A)<\infty$, we define its Morse index as the integer denoted by $\mu_{\mathrm{Mor}}[A]$ and defined as $\mu_{\text {Mor }}[A]:=\operatorname{dim} E_{-}(A)$. Given $A \in \mathcal{C F}^{s a}(\mathcal{H})$, for $a, b \notin \sigma(A)$ we set

$$
\mathcal{P}_{[a, b]}(A):=\operatorname{Re}\left(\frac{1}{2 \pi i} \int_{\gamma}(\lambda-A)^{-1} d \lambda\right)
$$

where $\gamma$ is the circle of radius $\frac{b-a}{2}$ around the point $\frac{a+b}{2}$. We recall that if $[a, b] \subset \sigma(A)$ consists of isolated eigenvalues of finite type then $\operatorname{ImP}_{[a, b]}(A)^{2}=E_{[a, b]}(A):=\bigoplus_{\lambda \in(a, b)} \operatorname{ker}(\lambda-A) ;(\mathrm{cf}$. [GGK90, Section XV.2], for instance) and 0 either belongs in the resolvent set of $A$ or it is an isolated eigenvalue of finite multiplicity. Let us now consider the graph distance topology which is the topology induced by the gap metric $d_{G}\left(A_{1}, A_{2}\right):=\left\|P_{1}-P_{2}\right\|$ where $P_{i}$ is the orthogonal projection onto the graph of $A_{i}$ in the product space $\mathcal{H} \times \mathcal{H}$. The next result allow us to define the spectral flow for gap continuous paths in $\mathcal{C F}^{s a}(\mathcal{H})$.

Proposition A.9. Let $A_{0} \in \mathcal{E F}^{s a}(\mathcal{H})$ be fixed.

(i) There exists a positive real number a $\notin \sigma\left(A_{0}\right)$ and an open neighborhood $\mathscr{N} \subset \mathcal{C F}^{s a}(\mathcal{H})$ of $A_{0}$ in the gap topology such that $\pm a \notin \sigma(A)$ for all $A \in \mathscr{N}$ and the map

$$
\mathscr{N} \ni A \longmapsto \mathcal{P}_{[-a, a]}(A) \in \mathscr{L}(\mathcal{H})
$$

is continuous and the projection $\mathcal{P}_{[-a, a]}(A)$ has constant finite rank for all $t \in \mathscr{N}$.

(ii) If $\mathscr{N}$ is a neighborhood as in (i) and $-a \leq c \leq d \leq a$ are such that $c, d \notin \sigma(A)$ for all $A \in \mathscr{N}$, then $A \mapsto \mathcal{P}_{[c, d]}(A)$ is continuous on $\mathscr{N}$. Moreover the rank of $\mathcal{P}_{[c, d]}(A) \in \mathscr{N}$ is finite and constant.

Proof. For the proof of this result we refer the interested reader to [BLP05, Proposition 2.10].

Let $\mathcal{A}:[a, b] \rightarrow \mathcal{C F}^{s a}(\mathcal{H})$ be a gap continuous path. As consequence of Proposition A.9, for every $t \in[a, b]$ there exists $a>0$ and an open connected neighborhood $\mathscr{N}_{t, a} \subset \mathcal{C} \mathcal{F}^{s a}(\mathcal{H})$ of $\mathcal{A}(t)$ such that $\pm a \notin \sigma(A)$ for all $A \in \mathscr{N}_{t, a}$ and the map $\mathscr{N}_{t, a} \in A \longmapsto \mathcal{P}_{[-a, a]}(A) \in \mathcal{B}$ is continuous and hence rank $\left(\mathcal{P}_{[-a, a]}(A)\right)$ does not depends on $A \in \mathscr{N}_{t, a}$. Let us consider the open covering of the interval $[a, b]$ given by the pre-images of the neighborhoods $\mathcal{N}_{t, a}$ through $\mathcal{A}$ and, by choosing a sufficiently fine partition of the interval $[a, b]$ having diameter less than the Lebesgue number of the covering, we can find $a=: t_{0}<t_{1}<\cdots<t_{n}:=b$, operators $T_{i} \in \mathcal{C F}^{s a}(\mathcal{H})$ and positive real numbers $a_{i}, i=1, \ldots, n$ in such a way the restriction of the path $\mathcal{A}$ on the interval $\left[t_{i-1}, t_{i}\right]$ lies in the neighborhood $\mathscr{N}_{t_{i}, a_{i}}$ and hence the $\operatorname{dim} E_{\left[-a_{i}, a_{i}\right]}\left(\mathcal{A}_{t}\right)$ is constant for $t \in\left[t_{i-1}, t_{i}\right], i=1, \ldots, n$.

Definition A.10. The spectral flow of $\mathcal{A}$ (on the interval $[a, b]$ ) is defined by

$$
\operatorname{sf}(\mathcal{A},[a, b]):=\sum_{i=1}^{N} \operatorname{dim} E_{\left[0, a_{i}\right]}\left(\mathcal{A}_{t_{i}}\right)-\operatorname{dim} E_{\left[0, a_{i}\right]}\left(\mathcal{A}_{t_{i-1}}\right) \in \mathbb{Z} .
$$


(In shorthand Notation we denote $\operatorname{sf}(\mathcal{A},[a, b])$ simply by $\operatorname{sf}(\mathcal{A})$ if no confusion is possible). The spectral flow as given in Definition A.10 is well-defined (in the sense that it is independent either on the partition or on the $a_{i}$ ) and only depends on the continuous path $\mathcal{A}$. Here We list one of the useful properties of the spectral flow.

(Path Additivity) If $\mathcal{A}_{1}, \mathcal{A}_{2}:[a, b] \rightarrow \mathcal{C F}^{s a}(\mathcal{H})$ are two continuous path such that $\mathcal{A}_{1}(b)=$ $\mathcal{A}_{2}(a)$, then sf $\left(\mathcal{A}_{1} * \mathcal{A}_{2}\right)=\operatorname{sf}\left(\mathcal{A}_{1}\right)+\operatorname{sf}\left(\mathcal{A}_{2}\right)$.

As already observed, the spectral flow, in general, depends on the whole path and not just on the ends. However, if the path has a special form, it actually depends on the end-points. More precisely, let $\mathcal{A}, \mathcal{B} \in \mathcal{C F}^{s a}(\mathcal{H})$ and let $\widetilde{\mathcal{A}}:[a, b] \rightarrow \mathcal{C F}^{s a}(\mathcal{H})$ be the path pointwise defined by $\widetilde{\mathcal{A}}(t):=\mathcal{A}+\widetilde{\mathcal{B}}(t)$ where $\widetilde{\mathcal{B}}$ is any continuous curve of $\mathcal{A}$-compact operators parametrised on $[a, b]$ such that $\widetilde{\mathcal{B}}(a):=0$ and $\widetilde{\mathcal{B}}(b):=\mathcal{B}$. In this case, the spectral flow depends of the path $\widetilde{A}$, only on the endpoints (cfr. [ZL99] and reference therein).

REMARK A.11. It is worth noticing that, since every operator $\widetilde{\mathcal{A}}(t)$ is a compact perturbation of a a fixed one, the path $\widetilde{\mathcal{A}}$ is actually a continuous path into $\mathscr{L}(\mathcal{W} ; \mathcal{H})$, where $\mathcal{W}:=\mathcal{D}(\mathcal{A})$.

Definition A.12. ([ZL99, Definition 2.8]). Let $\mathcal{A}, \mathcal{B} \in \operatorname{CF}^{s a}(\mathcal{H})$ and we assume that $\mathcal{B}$ is $\mathcal{A}$ compact (in the sense specified above). Then the relative Morse index of the pair $\mathcal{A}, \mathcal{A}+\mathcal{B}$ is defined by $I(\mathcal{A}, \mathcal{A}+\mathcal{B})=-\operatorname{sf}(\widetilde{\mathcal{A}} ;[a, b])$ where $\widetilde{\mathcal{A}}:=\mathcal{A}+\widetilde{\mathcal{B}}(t)$ and where $\widetilde{\mathcal{B}}$ is any continuous curve parametrised on $[a, b]$ of $\mathcal{A}$-compact operators such that $\widetilde{\mathcal{B}}(a):=0$ and $\widetilde{\mathcal{B}}(b):=\mathcal{B}$.

In the special case in which the Morse index of both operators $\mathcal{A}$ and $\mathcal{A}+\mathcal{B}$ are finite, then

$$
I(\mathcal{A}, \mathcal{A}+\mathcal{B})=\mu_{\mathrm{Mor}}[\mathcal{A}+\mathcal{B}]-\mu_{\mathrm{Mor}}[\mathcal{A}]
$$

Let $\mathcal{W}, \mathcal{H}$ be separable Hilbert spaces with a dense and continuous inclusion $\mathcal{W} \hookrightarrow \mathcal{H}$ and let $\mathcal{A}:[a, b] \rightarrow \mathcal{C F}^{s a}(\mathcal{H})$ having fixed domain $\mathcal{W}$. We assume that $\mathcal{A}$ is a continuously differentiable path $\mathcal{A}:[a, b] \rightarrow \mathcal{C} \mathcal{F}^{s a}(\mathcal{H})$ and we denote by $\dot{\mathcal{A}}_{\lambda_{0}}$ the derivative of $\mathcal{A}_{\lambda}$ with respect to the parameter $\lambda \in[a, b]$ at $\lambda_{0}$.

Definition A.13. An instant $\lambda_{0} \in[a, b]$ is called a crossing instant if ker $\mathcal{A}_{\lambda_{0}} \neq 0$. The crossing form at $\lambda_{0}$ is the quadratic form defined by

$$
\Gamma\left(\mathcal{A}, \lambda_{0}\right): \operatorname{ker} \mathcal{A}_{\lambda_{0}} \rightarrow \mathbb{R}, \quad \Gamma\left(\mathcal{A}, \lambda_{0}\right)[u]=\left\langle\dot{\mathcal{A}}_{\lambda_{0}} u, u\right\rangle_{\mathcal{H}}
$$

Moreover a crossing $\lambda_{0}$ is called regular, if $\Gamma\left(\mathcal{A}, \lambda_{0}\right)$ is non-degenerate.

We recall that there exists $\varepsilon>0$ such that $\mathcal{A}+\delta I \mathcal{H}$ has only regular crossings for almost every $\delta \in(-\varepsilon, \varepsilon)$. In the special case in which all crossings are regular, then the spectral flow can be easily computed through the crossing forms. More precisely the following result holds.

Proposition A.14. If $\mathcal{A}:[a, b] \rightarrow \mathcal{C F}^{s a}(\mathcal{W}, \mathcal{H})$ has only regular crossings then they are in a finite number and

$$
\operatorname{sf}(\mathcal{A},[a, b])=-\mathrm{n}_{-}[\Gamma(\mathcal{A}, a)]+\sum_{t_{0} \in(a, b)} \operatorname{sgn}\left[\Gamma\left(\mathcal{A}, t_{0}\right)\right]+\mathrm{n}_{+}[\Gamma(\mathcal{A}, b)]
$$

where the sum runs over all the crossing instants.

Proof. The proof of this result follows by arguing as in [RS95]. This conclude the proof.

\section{References}

[APS08] Abbondandolo, Alberto; Portaluri, Alessandro; Schwarz, Matthias The homology of path spaces and Floer homology with conormal boundary conditions. J. Fixed Point Theory Appl. 4 (2008), no. 2, 263-293.

[Ati84] Atiyah, Michael F. Anomalies and index theory. Lect. Notes in Physics 208 (1984), 313-322. 
[BJP14a] Barutello, Vivina; Jadanza D. Riccardo; Alessandro PorTALURI Linear instability of relative equilibria for $n$-body problems in the plane http://http://arxiv.org/abs/1310.8318. To appear in Journal of Differential Equations.

[BJP14b] Barutello, Vivina; Jadanza D. Riccardo; Alessandro Portaluri Morse index and linear stability of the Lagrangian circular orbit in a three-body-type problem via index theory In preparation

[BlP05] Booss-BavnbeK, Bernhelm; Lesch, Matthias; Phillips, John Unbounded Fredholm operators and spectral flow. Canad. J. Math. 57 (2005), no. 2, 225-250.

[Clm94] Cappell, Sylvain E.; Lee, Ronnie; Miller, Edward Y. On the Maslov index. Comm. Pure Appl. Math. 47 (1994), no. 2, 121-186.

[Dui76] DuistermaAt, J.J. On the Morse index in variational calculus. Adv. in Math. 21 (1976) no. $2,173-195$.

[GST96] Gesztesy, F.; Simon, B.; Teschl, G. Zeros of the Wronskian and renormalized oscillation theory. Amer. J. Math. 118, no. 3 (1996), 571-594.

[GGK90] I. Gohberg - S. Goldberg - M.Kaashoek. Classes of Linear operators, vol. 1 Birkauser, Verlag Basel Boston Berlin (1990).

[HP17] Hu, Xijun; Portaluri, Alessandro Index theory for heteroclinic orbits of Hamiltonian systems. Calc. Var. Partial Differential Equations 56 (2017), no. 6, Art. 167, 24 pp.

[HP19a] Hu, Xijun; Portaluri, Alessandro Bifurcation of heteroclinic orbits via an index theory. Math. Z. 292 (2019), no. 1-2, 705-723.

[HPY19] Hu, Xijun; Portaluri, Alessandro; Yang Ran Instability of semiRiemannian closed geodesics. To apper in Nonlinearity. Preprint available on https://arxiv.org/pdf/1706.07619.pdf

[HS09] Hu Xijun; Sun Shanzhong Index and Stability of Symmetric Periodic Orbits in Hamiltonian Systems with Application to Figure-Eight Orbit Commun. Math. Phys. 290 (2009), 737-777.

[HS10] Hu Xijun; Sun Shanzhong Morse index and stability of elliptic Lagrangian solutions in the planar three-body problem. Adv. Math. 223 (2010), no. 1, 98-119.

[HW16] Hu, Xijun; Wang, Penghui Eigenvalue problem of Sturm-Liouville systems with separated boundary conditions. Mathematische Zeitschrift, 283 (2016),1-2, 339-348.

[Kat80] Kato, Tosio Perturbation Theory for linear operators. Grundlehren der Mathematischen Wissenschaften, 132, Springer-Verlag (1980).

[KOP19] Kavle, Henry; Offin, Daniel; Portaluri, Alessandro Keplerian orbits through the Conley-Zehnder index Preprint available at https://arxiv.org/pdf/1908.00075.pdf

[Kli83] W. Klingenberg, Closed geodesics on Riemannian manifolds CBMS Regional Conference Series in Mathematics, 53 (1983).

[Lon02] Long, Yiming Index theory for symplectic paths with applications. Progress in Mathematics, 207. Birkhäuser Verlag, Basel, 2002.

[LT98] Lesch, Matthias; Tolksdorff,JÜrgen On the determinant of one dimensional elliptic boundary value problems. Comm. Math. Phys. 193, no. 3 (1998), 643-660.

[LZ00] Long, Yiming; Zhu, Chaofeng

Maslov-type index theory for symplectic paths and spectral flow. II. Chinese Ann. Math. Ser. B, 21.1 (2000), 89-108. 
[MN99] Magnus, Jan R.; Neudecker, Heinz Matrix differential calculus with applications in statistics and econometrics. Wiley Series in Probability and Statistics. John Wiley \& Sons, Ltd., Chichester, 1999.

[MPP05] Musso, M.; Pejsachowicz, J.; Portaluri, A. A Morse index theorem for perturbed geodesics on semi-Riemannian manifolds. Topol. Methods Nonlinear Anal. 25 (2005), no. 1, 69-99.

[MPP07] Musso, Monica; Pejsachowicz, Jacobo; Portaluri, Alessandro Morse index and bifurcation of p-geodesics on semi Riemannian manifolds. ESAIM Control Optim. Calc. Var. 13 (2007), no. 3, 598-621.

[Por09] Portaluri, Alessandro Indefinite Sturm theory. (Russian) Funktsional. Anal. i Prilozhen. 43 (2009), no. 4, 91-96; translation in Funct. Anal. Appl. 43 (2009), no. 4, 316-319.

[Por11] Portaluri, Alessandro A K-theoretical invariant and bifurcation for a parameterized family of functionals. J. Math. Anal. Appl. 377 (2011), no. 2, 762-770.

[PPT04] Piccione, Paolo; Portaluri, Alessandro; Tausk, Daniel V. Spectral flow, Maslov index and bifurcation of semi-Riemannian geodesics. Ann. Global Anal. Geom. 25 (2004), no. 2, 121-149.

[PW14a] Portaluri, Alessandro; Waterstraat, Nils On bifurcation for semilinear elliptic Dirichlet problems and the Morse-Smale index theorem. J. Math. Anal. Appl. 408 (2013), no. 2, 572-575.

[PW14b] Portaluri, Alessandro; Waterstraat, Nils On bifurcation for semilinear elliptic Dirichlet problems on geodesic balls. J. Math. Anal. Appl. 415 (2014), no. 1, 240-246.

[PW14c] Portaluri, Alessandro; Waterstraat, Nils Bifurcation results for critical points of families of functionals. Differential Integral Equations 27 (2014), no. 3-4, 369-386.

[PWY19] Portaluri, Alessandro; Wu, LI; YAng RAN Linear instability for periodic orbits of non-autonomous Lagrangian systems Preprint available at https://arxiv.org/pdf/1907.05864.pdf

[RS93] Robbin, Joel; Salamon, Dietmar The Maslov index for paths. Topology 32(1993), no.4, 827-844.

[RS95] Robbin, Joel; Salamon, Dietmar The spectral flow and the Maslov index. Bull. London Math. Soc. 27 (1995), no.1, 1-33.

[Sma65] Smale S. On the Morse index theorem. J. Math. Mech. 141965 1049-1055.

[Sma67] Smale S. Corrigendum: "On the Morse index theorem" J. Math. Mech. 1619671069 1070.

[ZL99] Zhu, Chaofeng; Long, Yiming Maslov-type index theory for symplectic paths and spectral flow. I. Chinese Ann. Math. Ser. B 20 (1999), no. 4, 413-424.

Prof. Alessandro Portaluri

DISAFA

Università degli Studi di Torino

Largo Paolo Braccini 2

10095 Grugliasco, Torino

Italy

Website: https://sites.google.com/view/alessandro-portaluri/

E-mail: alessandro.portaluri@unito.it 
Prof. Li Wu

Department of Mathematics

Shandong University

Jinan,Shandong, 250100

The People's Republic of China

China

E-mail: vvvli@sdu.edu.cn 